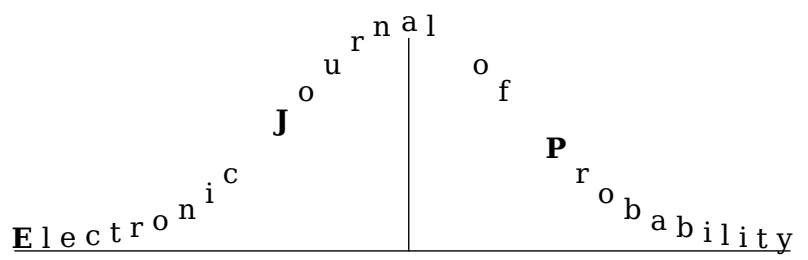

Electron. J. Probab. 25 (2020), no. 40, 1-31.

ISSN: 1083-6489 https://doi.org/10.1214/20-EJP438

\title{
Optimal lower bounds on hitting probabilities for stochastic heat equations in spatial dimension $k \geq 1^{*}$
}

\author{
Robert C. Dalang ${ }^{\dagger} \quad$ Fei $\mathrm{Pu}^{\ddagger}$
}

\begin{abstract}
We establish a sharp estimate on the negative moments of the smallest eigenvalue of the Malliavin matrix $\gamma_{Z}$ of $Z:=(u(s, y), u(t, x)-u(s, y))$, where $u$ is the solution to a system of $d$ non-linear stochastic heat equations in spatial dimension $k \geq 1$. We also obtain the optimal exponents for the $L^{p}$-modulus of continuity of the increments of the solution and of its Malliavin derivatives. These lead to optimal lower bounds on hitting probabilities of the process $\left\{u(t, x):(t, x) \in\left[0, \infty\left[\times \mathbb{R}^{k}\right\}\right.\right.$ in the non-Gaussian case in terms of Newtonian capacity, and improve a result in Dalang, Khoshnevisan and Nualart [Stoch PDE: Anal Comp 1 (2013) 94-151].
\end{abstract}

Keywords: hitting probabilities; systems of non-linear stochastic heat equations; spatially homogeneous Gaussian noise; Malliavin calculus.

AMS MSC 2010: 60H15; 60J45; 60H07; 60G60.

Submitted to EJP on November 30, 2018, final version accepted on February 28, 2020.

\section{Introduction and main results}

Consider the following system of stochastic partial differential equations:

$$
\frac{\partial}{\partial t} u_{i}(t, x)=\frac{1}{2} \Delta_{x} u_{i}(t, x)+\sum_{j=1}^{d} \sigma_{i j}(u(t, x)) \dot{F}^{j}(t, x)+b_{i}(u(t, x)),
$$

for $1 \leq i \leq d, t \in[0, T]$ and $x \in \mathbb{R}^{k}(k \geq 1)$, where $u:=\left(u_{1}, \ldots, u_{d}\right)$ with initial conditions $u(0, x)=0$ for all $x \in \mathbb{R}^{k}$, and the $\Delta_{x}$ denotes the Laplacian in the spatial variable $x$. The functions $\sigma_{i j}, b_{i}: \mathbb{R}^{d} \rightarrow \mathbb{R}$ are globally Lipschitz functions, $i, j \in\{1, \ldots, d\}$. We set $b=\left(b_{i}\right)$ and $\sigma=\left(\sigma_{i j}\right)$.

The noise $\dot{F}=\left(\dot{F}_{1}, \ldots, \dot{F}_{d}\right)$ is a spatially homogeneous centered Gaussian generalized random field with covariance of the form

$$
\mathrm{E}\left[\dot{F}^{i}(t, x) \dot{F}^{j}(s, y)\right]=\delta(t-s)\|x-y\|^{-\beta} \delta_{i j},
$$

${ }^{*}$ Research partially supported by the Swiss National Foundation for Scientific Research.

†École Polytechnique Fédérale de Lausanne. E-mail: robert.dalang@epfl.ch

‡École Polytechnique Fédérale de Lausanne. E-mail: fei.pu@epfl.ch 
where $\beta \in] 0,2 \wedge k\left[, \delta(\cdot)\right.$ denotes the Dirac delta function, $\delta_{i j}$ the Kronecker symbol and $\|\cdot\|$ is the Euclidean norm. In particular, the $d$-dimensional driving noise $\dot{F}$ is white in time and with a spatially homogeneous covariance given by the Riesz kernel $f(x)=\|x\|^{-\beta}$.

The solution $u$ of (1.1) is known to be a $d$-dimensional random field (see the end of this section where precise definitions and references are given). The potential theory for $u$ has been developed by Dalang, Khoshnevisan and Nualart [6]. Fix $T>0$ and let $I \times J \subset] 0, T] \times \mathbb{R}^{k}$ be a closed non-trivial rectangle. In the case where the noise is additive, i.e., $\sigma \equiv \mathrm{Id}$ and $b \equiv 0$, Dalang, Khoshnevisan and Nualart [6, Theorem 1.5] prove that for fixed $M>0$, there exists $c>0$ depending on $I, J$ and $M$ such that for all compact sets $A \subseteq[-M, M]^{d}$,

$$
\mathrm{P}\{u(I \times J) \cap A \neq \emptyset\} \geq c \operatorname{Cap}_{d-\left(\frac{4+2 k}{2-\beta}\right)}(A),
$$

where $u(I \times J)$ denotes the range of $I \times J$ under the random map $(t, x) \mapsto u(t, x)$, and $\operatorname{Cap}_{\beta}$ denotes the capacity with respect to the Newtonian $\beta$-kernel (we refer to [6, Section 1] for the definition of capacity). If the noise is multiplicative, i.e., $\sigma$ and $b$ are not constants (but are sufficiently regular), then using techniques of Malliavin calculus, Dalang, Khoshnevisan and Nualart [6, Theorem 1.2(b)] prove that for fixed $M>0$ and $\eta>0$, there exists $c>0$ depending on $I, J, M$ and $\eta$ such that for all compact sets $A \subseteq[-M, M]^{d}$,

$$
\mathrm{P}\{u(I \times J) \cap A \neq \emptyset\} \geq c \operatorname{Cap}_{d-\left(\frac{4+2 k}{2-\beta}\right)+\eta}(A) .
$$

For systems of linear and/or non-linear stochastic heat equations in spatial dimension 1 driven by a $d$-dimensional space-time white noise, this type of question was studied in Dalang, Khoshnevisan and Nualart [4] and [5], in which the lower bounds on hitting probabilities in the Gaussian case (additive noise) and non-Gaussian case (multiplicative noise) are not identical. This discrepancy has been filled recently by Dalang and $\mathrm{Pu}$ [7], in which we have obtained the optimal lower bounds on hitting probabilities for systems of non-linear stochastic heat equations in spatial dimension 1.

The aim of this paper is to remove the $\eta$ in the dimension of capacity in (1.4), so that we obtain the optimal lower bounds on hitting probabilities for systems of non-linear stochastic heat equations in higher spatial dimension.

In [6], the lower bound on the hitting probability in (1.4) follows from the properties of the probability density function of the solution (see [6, Theorems 1.6 and 1.8]), in particular, the upper bound on the joint probability density function (denoted by $p_{Z}(\cdot, \cdot)$ ) of the random vector

$$
Z:=(u(s, y), u(t, x)-u(s, y)) .
$$

In [6, Corollary 5.10], the formula for the density function $p_{Z}(\cdot, \cdot)$ is given in terms of the Malliavin derivative and the Skorohod integral (we refer to Section 2 for the elements of Malliavin calculus). From this formula, in order to establish an upper bound on the density function $p_{Z}(\cdot, \cdot)$, the main effort is to analyze the $L^{p}$-modulus of continuity of the increments of the solution (see [6, (2.6)]) and of the Malliavin derivative of the increments of the solution (see [6, Proposition 5.1]), and the negative moments of the smallest eigenvalue of the Malliavin matrix $\gamma_{Z}$ of $Z$ (see [6, Proposition 5.6]). We point out that the estimates in [6, (2.6), Propositions 5.1 and 5.6] are not sharp, and that is why the extra term $\eta$ appears in (1.4).

We first look at the $L^{p}$-modulus of continuity of the increments of the solution. Hölder continuity for the solution to stochastic heat equation with spatially correlated noise has been studied by many authors; see, for example, [8, 9, 17]. Sanz-Solé and Sarrà [17] use the factorization method to study the Hölder continuity for the solution to (1.1) (with $d=1$ ), when the initial condition is bounded and $\delta$-Hölder continuous for some 
$\delta \in] 0,1[$, and the spatial covariance of the noise $\dot{F}$ is the Fourier transform of a tempered measure $\mu$ on $\mathbb{R}^{k}$. In particular, [17, Theorem 2.1] shows that, if the measure $\mu$ satisfies the condition

$$
\left.\int_{\mathbb{R}^{k}} \frac{\mu(d \xi)}{\left(1+\|\xi\|^{2}\right)^{\eta}}<\infty, \text { for some } \eta \in\right] 0,1[,
$$

then for any $p \geq 2$ and $\gamma \in] 0, \delta \wedge(1-\eta)$ [, there exists $C(p, T)>0$ such that for all $(t, x)$, $(s, y) \in[0, T] \times \mathbb{R}^{k}$,

$$
\mathrm{E}\left[|u(t, x)-u(s, y)|^{p}\right] \leq C(p, T)\left(|t-s|^{\gamma / 2}+\|x-y\|^{\gamma}\right)^{p}
$$

see [17, (10) and (19)]. In the case where $f=\mathscr{F} \mu$ is the Riesz kernel $f(x)=\|x\|^{-\beta}$ and the initial value vanishes, this result of Sanz-Solé and Sarrà becomes: for any $\gamma \in] 0, \frac{2-\beta}{2}[$,

$$
\mathrm{E}\left[|u(t, x)-u(s, y)|^{p}\right] \leq C(p, T)\left(|t-s|^{\gamma / 2}+\|x-y\|^{\gamma}\right)^{p}
$$

for all $(t, x),(s, y) \in[0, T] \times \mathbb{R}^{k}$. Note that the right endpoint $\gamma=\frac{2-\beta}{2}$ is excluded.

Li [9] has studied the Hölder continuity for stochastic fractional heat equations without drift in the case where the Gaussian noise is white in time and colored in space with covariance of the form (1.2). Based on some estimates of the fractional heat kernel, [9, Theorems 1, 2 and 3] obtains spatial and temporal $L^{p}$-Hölder continuity of the solution to stochastic fractional heat equation. In these results, the exponent in time is optimal while the exponent in space is not ([9, Remark 2]).

The first contribution of this paper is the following sharp estimate of the $L^{p}$-Hölder continuity for the solution to (1.1), improving (1.6). We have the following.

Theorem 1.1. Assume that $\sigma_{i j}$ and $b_{i}$ are globally Lipschitz continuous. There exists a constant $C_{p, T}>0$ such that for all $s, t \in[0, T], s \leq t, x, y \in \mathbb{R}^{k}, p \geq 2$,

$$
\mathrm{E}\left[\|u(t, x)-u(s, y)\|^{p}\right] \leq C_{p, T}\left(|t-s|^{(2-\beta) / 4}+\|x-y\|^{(2-\beta) / 2}\right)^{p} .
$$

We also need the $L^{p}$-Hölder continuity for the Malliavin derivative of the solution to (1.1). We consider the following hypotheses on the coefficients of the system (1.1):

P1 The functions $\sigma_{i j}$ and $b_{i}$ are infinitely differentiable with bounded partial derivatives of all positive orders, and the $\sigma_{i j}$ are bounded, for $1 \leq i, j \leq d$.

P2 The matrix $\sigma$ is uniformly elliptic, that is, $\|\sigma(x) \xi\|^{2} \geq \rho^{2}>0$ for some $\rho>0$, for all $x \in \mathbb{R}^{d},\|\xi\|=1$.

Analogous to Theorem 1.1, we have the following sharp estimate of the $L^{p}$-Hölder continuity for the Malliavin derivative of the solution to (1.1), which is an improvement of [6, Proposition 5.1].

Theorem 1.2. Assume P1. Then for any $T>0$ and $p \geq 2$, there exists a constant $C:=C_{p, T}>0$ such that for any $0 \leq s \leq t \leq T, x, y \in \mathbb{R}^{k}, m \geq 1$ and $i \in\{1, \ldots, d\}$,

$$
\left\|D^{m}\left(u_{i}(t, x)-u_{i}(s, y)\right)\right\|_{L^{p}\left(\Omega ;\left(\mathscr{H}_{T}^{d}\right) \otimes m\right.} \leq C\left(|t-s|^{\frac{2-\beta}{4}}+\|x-y\|^{\frac{2-\beta}{2}}\right) .
$$

Theorems 1.1 and 1.2 are proved in Section 4. Turning to the negative moments of the smallest eigenvalue of the Malliavin matrix $\gamma_{Z}$ of the random vector $Z$ (defined in (1.5)), we have the following sharp estimate, which is an improvement of [6, Proposition $5.6]$. 
Theorem 1.3. Assume P1 and P2. Fix $T>0$ and let $I \times J \subset] 0, T] \times \mathbb{R}^{k}$ be a closed non-trivial rectangle (that is, $I$ is a non-trivial interval and $J=J_{1} \times \cdots \times J_{n}$, where $J_{1}, \ldots, J_{k}$ are non-trivial intervals). There exists $C>0$ depending on $T$ such that for all $s, t \in I, 0 \leq t-s<1, x, y \in J,(s, y) \neq(t, x)$, and $p>1$,

$$
\mathrm{E}\left[\left(\inf _{\substack{\xi=(\lambda, \mu) \in \mathbb{R}^{2 d}: \\\|\lambda\|^{2}+\|\mu\|^{2}=1}} \xi^{T} \gamma_{Z} \xi\right)^{-2 d p}\right] \leq C\left(|t-s|^{\frac{2-\beta}{2}}+\|x-y\|^{2-\beta}\right)^{-2 d p} .
$$

Theorem 1.3 is proved in Section 5. Using Theorems 1.1, 1.2 and 1.3 and some results of [6], we establish a sharp upper bound on the joint probability density function (denoted by $\left.p_{s, y ; t, x}(\cdot, \cdot)\right)$ of the random vector $(u(s, y), u(t, x))$ and the optimal lower bounds on hitting probabilities of the solution to (1.1).

Theorem 1.4. Assume P1 and P2. Fix $T>0$ and let $I \times J \subset] 0, T] \times \mathbb{R}^{k}$ be a closed nontrivial rectangle. There exists $c>0$ such that for all $s, t \in I, x, y \in J$ with $(s, y) \neq(t, x)$, $z_{1}, z_{2} \in \mathbb{R}^{d}$ and $p \geq 1$,

$$
p_{s, y ; t, x}\left(z_{1}, z_{2}\right) \leq c\left[|t-s|^{\frac{2-\beta}{4}}+\|x-y\|^{\frac{2-\beta}{2}}\right]^{-d}\left[\frac{\left(|t-s|^{\frac{2-\beta}{4}}+\|x-y\|^{\frac{2-\beta}{2}}\right)^{2}}{\left\|z_{1}-z_{2}\right\|^{2}} \wedge 1\right]^{\frac{p}{2 d}} .
$$

Theorem 1.5. Assume P1 and P2. Fix $T>0$ and $M>0$. Let $I \times J \subset] 0, T] \times \mathbb{R}^{k}$ be a closed non-trivial rectangle. There exists $c>0$ depending on $I, J$ and $M$ such that for all compact sets $A \subseteq[-M, M]^{d}$,

$$
\mathrm{P}\{u(I \times J) \cap A \neq \emptyset\} \geq c \operatorname{Cap}_{\mathrm{d}-\left(\frac{4+2 \mathrm{k}}{2-\beta}\right)}(\mathrm{A}) .
$$

Theorem 1.4 is an improvement of [6, Theorem 1.6(b)] and Theorem 1.5 is an improvement of [6, Theorem 1.2(b)], and they are proved in Section 3. The main ingredients which allow for these improvements are the sharp $L^{p}$-Hölder continuity estimates of Theorems 1.1 and 1.2 and a better estimate on the Malliavin derivative of $u(t, x)$ given in Lemma 5.3 below.

We conclude this section by giving a rigorous formulation of (1.1), following Walsh [18]. We first define precisely the driving noise that appears in (1.1). Let "." denote the temporal variable and "*" the spatial variable. Let $\mathscr{D}\left(\mathbb{R}^{k+1}\right)$ be the space of $C^{\infty}$ testfunctions with compact support. Then $F=\left\{F(\phi)=\left(F^{1}(\phi), \ldots, F^{d}(\phi)\right), \phi \in \mathscr{D}\left(\mathbb{R}^{k+1}\right)\right\}$ is an $L^{2}(\Omega, \mathscr{F}, \mathrm{P})^{d}$-valued mean zero Gaussian process with covariance

$$
\mathrm{E}\left[F^{i}(\phi) F^{j}(\psi)\right]=\delta_{i j} \int_{\mathbb{R}_{+}} d r \int_{\mathbb{R}^{k}} d y \int_{\mathbb{R}^{k}} d z \phi(r, y)\|y-z\|^{-\beta} \psi(r, z) .
$$

Using elementary properties of the Fourier transform (see Dalang [2]), this covariance can also be written as

$$
\mathrm{E}\left[F^{i}(\phi) F^{j}(\psi)\right]=\delta_{i j} c_{k, \beta} \int_{\mathbb{R}_{+}} d r \int_{\mathbb{R}^{k}} d \xi\|\xi\|^{\beta-k} \mathscr{F} \phi(r, *)(\xi) \overline{\mathscr{F} \psi(r, *)(\xi)},
$$

where $c_{k, \beta}$ is a constant and $\mathscr{F} f(\xi)$ is the Fourier transform of $f$, that is,

$$
\mathscr{F} f(\xi)=\int_{\mathbb{R}^{k}} e^{-i \xi \cdot x} f(x) d x .
$$

Following Walsh [18], a rigorous formulation of (1.1) through the notion of mild solution is as follows. Let $M=\left(M^{1}, \ldots, M^{d}\right), M^{i}=\left\{M_{t}^{i}(A), t \geq 0, A \in \mathscr{B}_{b}\left(\mathbb{R}^{k}\right)\right\}$ be the 
$d$-dimensional worthy martingale measure obtained as an extension of the process $\dot{F}$ as in Dalang and Frangos [3], so that the covariation measure of $M$ is

$$
Q(A \times B \times] s, t])=(t-s) \int_{A} d y \int_{B} d z\|y-z\|^{-\beta}
$$

and the dominating measure of $M$ is $K=Q$. Then a mild solution of (1.1) is a jointly measurable $\mathbb{R}^{d}$-valued process $u=\left\{u(t, x), t \geq 0, x \in \mathbb{R}^{k}\right\}$, adapted to the natural filtration generated by $M$, such that

$$
\begin{aligned}
u_{i}(t, x)= & \int_{0}^{t} \int_{\mathbb{R}^{k}} S(t-s, x-y) \sum_{j=1}^{d} \sigma_{i j}(u(s, y)) M^{j}(d s, d y) \\
& +\int_{0}^{t} d s \int_{\mathbb{R}^{k}} d y S(t-s, x-y) b_{i}(u(s, y)), \quad i \in\{1, \ldots, d\},
\end{aligned}
$$

where $S(t, x)$ is the fundamental solution of the deterministic heat equation in $\mathbb{R}^{k}$, that is,

$$
S(t, x)=(2 \pi t)^{-k / 2} \exp \left(-\frac{\|x\|^{2}}{2 t}\right),
$$

and the stochastic integral is interpreted in the sense of [18].

Using the results of Dalang [2], existence and uniqueness of the solution of (1.1) holds, as discussed in [6, Section 2], under the condition

$$
0<\beta<(2 \wedge k)
$$

and in this case, there exists a unique $L^{2}$-continuous solution of (1.12) satisfying

$$
\sup _{(t, x) \in[0, T] \times \mathbb{R}^{k}} \mathrm{E}\left[\left|u_{i}(t, x)\right|^{p}\right]<\infty, \quad i \in\{1, \ldots, d\},
$$

for any $T>0$ and $p \geq 1$.

\section{Elements of Malliavin calculus}

In this section, we introduce, following Nualart [11] (see also [16]), some elements of Malliavin calculus. Let $\mathscr{S}\left(\mathbb{R}^{k}\right)$ be the Schwartz space of $C^{\infty}$ functions on $\mathbb{R}^{k}$ with rapid decrease. Let $\mathscr{H}$ denote the completion of $\mathscr{S}\left(\mathbb{R}^{k}\right)$ endowed with their inner product

$$
\begin{aligned}
\langle\phi, \psi\rangle_{\mathscr{H}} & =\int_{\mathbb{R}^{k}} d x \int_{\mathbb{R}^{k}} d y \phi(x)\|x-y\|^{-\beta} \psi(y) \\
& =c_{k, \beta} \int_{\mathbb{R}^{k}} d \xi\|\xi\|^{\beta-k} \mathscr{F} \phi(\xi) \overline{\mathscr{F}} \psi(\xi)
\end{aligned}
$$

$\phi, \psi \in \mathscr{S}\left(\mathbb{R}^{k}\right)$.

For $h=\left(h_{1}, \ldots, h_{d}\right) \in \mathscr{H}^{d}$ and $\tilde{h}=\left(\tilde{h}_{1}, \ldots, \tilde{h}_{d}\right) \in \mathscr{H}^{d}$, we set $\langle h, \tilde{h}\rangle_{\mathscr{C}^{d}}=\sum_{i=1}^{d}\left\langle h_{i}, \tilde{h}_{i}\right\rangle_{\mathscr{H}}$. Let $T>0$ be fixed. We set $\mathscr{H}_{T}^{d}=L^{2}\left([0, T] ; \mathscr{H}^{d}\right)$ and for $0 \leq s \leq t \leq T$, we will write $\mathscr{H}_{s, t}^{d}=L^{2}\left([s, t], \mathscr{H}^{d}\right)$.

The centered Gaussian noise $F$ can be used to construct an isonormal Gaussian process $\left\{W(h), h \in \mathscr{H}_{T}^{d}\right\}$ as follows. Let $\left\{e_{j}, j \geq 0\right\} \subset \mathscr{S}\left(\mathbb{R}^{k}\right)$ be a complete orthonormal system of the Hilbert space $\mathscr{H}$. Then for any $t \in[0, T], i \in\{1, \ldots, d\}$ and $j \geq 0$, set

$$
W_{j}^{i}(t)=\int_{0}^{t} \int_{\mathbb{R}^{k}} e_{j}(x) \cdot F^{i}(d s, d x)
$$


so that $\left(W_{j}^{i}, j \geq 1\right)$ is a sequence of independent standard real-valued Brownian motions such that, for any $\phi \in \mathscr{D}\left([0, T] \times \mathbb{R}^{k}\right)$,

$$
F^{i}(\phi)=\sum_{j=0}^{\infty} \int_{0}^{T}\left\langle\phi(s, *), e_{j}(*)\right\rangle_{\mathscr{H}} d W_{j}^{i}(s)
$$

where the series converges in $L^{2}(\Omega, \mathscr{F}, \mathrm{P})$. For $h^{i} \in \mathscr{H}_{T}$, we set

$$
W^{i}(h)=\sum_{j=0}^{\infty} \int_{0}^{T}\left\langle h^{i}(s, *), e_{j}(*)\right\rangle_{\mathscr{H}} d W_{j}^{i}(s)
$$

where, again, the series converges in $L^{2}(\Omega, \mathscr{F}, \mathrm{P})$. In particular, for $\phi \in \mathscr{D}\left([0, T] \times \mathbb{R}^{k}\right)$, $F^{i}(\phi)=W^{i}(\phi)$. Finally, for $h=\left(h_{1}, \ldots, h_{d}\right) \in \mathscr{H}_{T}^{d}$, we set

$$
W(h)=\sum_{i=1}^{d} W^{i}\left(h^{i}\right) .
$$

With this isonormal Gaussian process, we can use the framework of Malliavin calculus. Let $\mathcal{S}$ denote the class of smooth random variables of the form

$$
G=g\left(W\left(h_{1}\right), \ldots, W\left(h_{n}\right)\right),
$$

where $n \geq 1, g \in \mathscr{C}_{p}^{\infty}\left(\mathbb{R}^{n}\right)$, the set of real-valued functions $g$ such that $g$ and all its partial derivatives have at most polynomial growth and $h_{i} \in \mathscr{H}_{T}^{d}$. Given $G \in \mathcal{S}$, its derivative $\left(D_{r} G=\left(D_{r}^{(1)} G, \ldots, D_{r}^{(d)} G\right), r \in[0, T]\right)$ is an $\mathscr{H}_{T}^{d}$-valued random vector defined by

$$
D_{r} G=\sum_{i=1}^{n} \partial_{i} g\left(W\left(h_{1}\right), \ldots, W\left(h_{n}\right)\right) h_{i}(r) .
$$

For $\phi \in \mathscr{H}^{d}$ and $r \in[0, T]$, we write $D_{r, \phi} G=\left\langle D_{r} G, \phi(*)\right\rangle_{\mathscr{C}^{d}}$. More generally, the derivative $D^{m} G=\left(D_{\left(r_{1}, \ldots, r_{m}\right)}^{m},\left(r_{1}, \ldots, r_{m}\right) \in[0, T]^{m}\right)$ of order $m \geq 1$ of $G$ is the $\left(\mathscr{H}_{T}^{d}\right)^{\otimes m}$ valued random vector defined by

$$
D_{\left(r_{1}, \ldots, r_{m}\right)}^{m} G=\sum_{i_{1}, \ldots, i_{m}=1}^{n} \frac{\partial}{\partial x_{i_{1}}} \cdots \frac{\partial}{\partial x_{i_{m}}} g\left(W\left(h_{1}\right), \ldots, W\left(h_{n}\right)\right) h_{i_{1}}\left(r_{1}\right) \otimes \cdots \otimes h_{i_{m}}\left(r_{m}\right),
$$

where the notation $\otimes$ denotes the tensor product of functions.

For $p, m \geq 1$, the space $\mathbb{D}^{m, p}$ is the closure of $\mathcal{S}$ with respect to the seminorm $\|\cdot\|_{m, p}$ defined by

$$
\|G\|_{m, p}^{p}=\mathrm{E}\left[|G|^{p}\right]+\sum_{j=1}^{m} \mathrm{E}\left[\left\|D^{j} G\right\|_{\left(\mathscr{H}_{T}^{d}\right) \otimes j}^{p}\right] .
$$

We set $\mathbb{D}^{\infty}=\cap_{p \geq 1} \cap_{m \geq 1} \mathbb{D}^{m, p}$.

The derivative operator $D$ on $L^{2}(\Omega)$ has an adjoint, termed the Skorohod integral and denoted by $\delta$, which is an unbounded and closed operator on $L^{2}\left(\Omega, \mathscr{H}_{T}^{d}\right)$; see [11, Section 1.3]. Its domain, denoted by $\operatorname{Dom} \delta$, is the set of elements $u \in L^{2}\left(\Omega, \mathscr{H}_{T}^{d}\right)$ such that there exists a constant $c$ such that $\left|\mathrm{E}\left[\langle D G, u\rangle_{\mathscr{H}_{T}^{d}}\right]\right| \leq c\|G\|_{0,2}$, for any $G \in \mathbb{D}^{1,2}$. If $u \in \operatorname{Dom} \delta$, then $\delta(u)$ is the element of $L^{2}(\Omega)$ characterized by the following duality relation:

$$
\mathrm{E}[G \delta(u)]=\mathrm{E}\left[\langle D G, u\rangle_{\mathscr{H}_{T}^{d}}\right], \text { for all } G \in \mathbb{D}^{1,2} .
$$


Recall from [6, Section 3] that for $r \in[0, t]$ and $i, l \in\{1, \ldots, d\}$, the derivative of $u_{i}(t, x)$ satisfies the system of equations

$$
D_{r}^{(l)}\left(u_{i}(t, x)\right)=\sigma_{i l}(u(r, *)) S(t-r, x-*)+a_{i}(l, r, t, x),
$$

where

$$
\begin{aligned}
a_{i}(l, r, t, x)= & \int_{r}^{t} \int_{\mathbb{R}^{k}} S(t-\theta, x-\eta) \sum_{j=1}^{d} D_{r}^{(l)}\left(\sigma_{i j}(u(\theta, \eta))\right) M^{j}(d \theta, d \eta) \\
& +\int_{r}^{t} d \theta \int_{\mathbb{R}^{k}} d \eta S(t-\theta, x-\eta) D_{r}^{(l)}\left(b_{i}(u(\theta, \eta))\right),
\end{aligned}
$$

and $D_{r}^{(l)}\left(u_{i}(t, x)\right)=0$ if $r>t$. Moreover, by [13, Proposition 6.1], for any $p>1, m \geq 1$ and $i \in\{1, \ldots, d\}$, the order $m$ derivative satisfies

$$
\sup _{(t, x) \in[0, T] \times \mathbb{R}^{k}} \mathrm{E}\left[\left\|D^{m}\left(u_{i}(t, x)\right)\right\|_{\left(\mathscr{H}_{T}^{d}\right) \otimes m}^{p}\right]<\infty,
$$

and $D^{m}$ also satisfies the system of stochastic partial differential equations given in [13, (6.29)] and obtained by iterating the calculation that leads to (2.1). In particular, $u(t, x) \in\left(\mathbb{D}^{\infty}\right)^{d}$, for all $(t, x) \in[0, T] \times \mathbb{R}^{k}$.

\section{Proof of Theorems 1.4 and 1.5 (assuming Theorems 1.1-1.3)}

Recall that the Malliavin matrix $\gamma_{Z}$ of $Z=(u(s, y), u(t, x)-u(s, y))$ is a symmetric $2 d \times 2 d$ random matrix with four $d \times d$ blocs of the form

$$
\gamma_{Z}=\left(\begin{array}{ccc}
\gamma_{Z}^{(1)} & \vdots & \gamma_{Z}^{(2)} \\
\cdots & \vdots & \cdots \\
\gamma_{Z}^{(3)} & \vdots & \gamma_{Z}^{(4)}
\end{array}\right)
$$

where

$$
\begin{aligned}
& \gamma_{Z}^{(1)}=\left(\left\langle D\left(u_{i}(s, y)\right), D\left(u_{j}(s, y)\right)\right\rangle_{\mathscr{H}_{T}^{d}}\right)_{i, j=1, \ldots, d}, \\
& \gamma_{Z}^{(2)}=\left(\left\langle D\left(u_{i}(s, y)\right), D\left(u_{j}(t, x)-u_{j}(s, y)\right)\right\rangle_{\mathscr{H}_{T}^{d}}\right)_{i, j=1, \ldots, d}, \\
& \gamma_{Z}^{(3)}=\left(\left\langle D\left(u_{i}(t, x)-u_{i}(s, y)\right), D\left(u_{j}(s, y)\right)\right\rangle_{\mathscr{H}_{T}^{d}}\right)_{i, j=1, \ldots, d}, \\
& \gamma_{Z}^{(4)}=\left(\left\langle D\left(u_{i}(t, x)-u_{i}(s, y)\right), D\left(u_{j}(t, x)-u_{j}(s, y)\right)\right\rangle_{\mathscr{H}_{T}^{d}}\right)_{i, j=1, \ldots, d} .
\end{aligned}
$$

We let (1) denote the couples of $\{1, \ldots, d\} \times\{1, \ldots, d\},(2)$ denote the couples of $\{1, \ldots, d\} \times$ $\{d+1, \ldots, 2 d\},(3)$ denote the couples of $\{d+1, \ldots, 2 d\} \times\{1, \ldots, d\}$ and (4) denote the couples of $\{d+1, \ldots, 2 d\} \times\{d+1, \ldots, 2 d\}$.

We first state two results which follow exactly along the same lines as [6, Propositions 5.3 and 5.4], using (2.3) and our Theorem 1.2 instead of their [6, (3.2) and Proposition 5.1]. Their proofs are omitted.

Proposition 3.1. Fix $T>0$ and let $I \times J \subset] 0, T] \times \mathbb{R}^{k}$ be a closed non-trivial rectangle. Let $A_{Z}$ denote the cofactor matrix of $\gamma_{Z}$. Assuming P1, for any $(s, y),(t, x) \in I \times J$, $(s, y) \neq(t, x), p>1$,

$$
\mathrm{E}\left[\left|\left(A_{Z}\right)_{m, l}\right|^{p}\right]^{1 / p} \leq \begin{cases}c_{p, T}\left(|t-s|^{\frac{2-\beta}{4}}+\|x-y\|^{\frac{2-\beta}{2}}\right)^{2 d} & \text { if }(m, l) \in(\mathbf{1}), \\ c_{p, T}\left(|t-s|^{\frac{2-\beta}{4}}+\|x-y\|^{\frac{2-\beta}{2}}\right)^{2 d-1} & \text { if }(m, l) \in(\mathbf{2}) \text { or }(\mathbf{3}), \\ c_{p, T}\left(|t-s|^{\frac{2-\beta}{4}}+\|x-y\|^{\frac{2-\beta}{2}}\right)^{2 d-2} & \text { if }(m, l) \in(\mathbf{4}) .\end{cases}
$$


Proposition 3.2. Fix $T>0$ and let $I \times J \subset] 0, T] \times \mathbb{R}^{k}$ be a closed non-trivial rectangle. Assuming P1, for any $(s, y),(t, x) \in I \times J,(s, y) \neq(t, x), p>1$,

$$
\mathrm{E}\left[\left\|D^{k}\left(\gamma_{Z}\right)_{m, l}\right\|_{\mathscr{H} \otimes k}^{p}\right]^{1 / p} \leq \begin{cases}c_{k, p, T} & \text { if }(m, l) \in(\mathbf{1}), \\ c_{k, p, T}\left(|t-s|^{\frac{2-\beta}{4}}+\|x-y\|^{\frac{2-\beta}{2}}\right) & \text { if }(m, l) \in(\mathbf{2}) \text { or }(\mathbf{3}), \\ c_{k, p, T}\left(|t-s|^{\frac{2-\beta}{4}}+\|x-y\|^{\frac{2-\beta}{2}}\right)^{2} & \text { if }(m, l) \in(\mathbf{4}) .\end{cases}
$$

The next result is an improvement of [6, Proposition 5.5].

Proposition 3.3. Fix $T>0$ and let $I \times J \subset] 0, T] \times \mathbb{R}^{k}$ be a closed non-trivial rectangle. Assume $\mathbf{P 1}$ and P2. There exists $C$ depending on $T$ such that for any $(s, y),(t, x) \in I \times J$, $(s, y) \neq(t, x), p>1$,

$$
\mathrm{E}\left[\left(\operatorname{det} \gamma_{Z}\right)^{-p}\right]^{1 / p} \leq C\left(|t-s|^{\frac{2-\beta}{4}}+\|x-y\|^{\frac{2-\beta}{2}}\right)^{-2 d} .
$$

Proof. Similar to the proof of [6, Proposition 5.5] (see also [5, Proposition 6.6]), this is a consequence of [6, (5.11)], our Theorem 1.3 and [6, Proposition 5.7].

From Propositions 3.1-3.3, we obtain the following result, which improves [6, Theorem 5.8]. The proof is similar to that of [6, Theorem 5.8] (but using our Proposition 3.3 instead of [6, Proposition 5.5]) and hence is omitted.

Proposition 3.4. Fix $T>0$ and let $I \times J \subset] 0, T] \times \mathbb{R}^{k}$ be a closed non-trivial rectangle. Assume P1 and P2. For any $(s, y),(t, x) \in I \times J,(s, y) \neq(t, x), k \geq 0$ and $p>1$,

$$
\mathrm{E}\left[\left\|\left(\gamma_{Z}\right)_{m, l}^{-1}\right\|_{k, p}\right] \leq \begin{cases}c_{k, p, T} & \text { if }(m, l) \in(\mathbf{1}), \\ c_{k, p, T}\left(|t-s|^{\frac{2-\beta}{4}}+\|x-y\|^{\frac{2-\beta}{2}}\right)^{-1} & \text { if }(m, l) \in(\mathbf{2}) \text { or }(\mathbf{3}), \\ c_{k, p, T}\left(|t-s|^{\frac{2-\beta}{4}}+\|x-y\|^{\frac{2-\beta}{2}}\right)^{-2} & \text { if }(m, l) \in(\mathbf{4}) .\end{cases}
$$

We are now ready to prove Theorems 1.4 and 1.5.

Proof of Theorem 1.4. We recall from the proof of [6, Theorem 1.6(b)] that

$$
p_{s, y ; t, x}\left(z_{1}, z_{2}\right)=p_{Z}\left(z_{1}, z_{2}-z_{1}\right) \text {, for all } z_{1}, z_{2} \in \mathbb{R}^{d},
$$

and

$$
\begin{aligned}
p_{Z}\left(z_{1}, z_{2}-z_{1}\right) \leq & \prod_{i=1}^{d}\left(\mathrm{P}\left\{\left|u_{i}(t, x)-u_{i}(s, y)\right|>\left|z_{1}^{i}-z_{2}^{i}\right|\right\}\right)^{\frac{1}{2 d}} \\
& \times\left\|H_{(1, \ldots, 2 d)}(Z, 1)\right\|_{0,2},
\end{aligned}
$$

where the random variable $H_{(1, \ldots, 2 d)}(Z, 1)$ is given by the formula in [6, Corollary 5.10]. Using Chebyshev's inequality and Theorem 1.1, we see that

$$
\prod_{i=1}^{d}\left(\mathrm{P}\left\{\left|u_{i}(t, x)-u_{i}(s, y)\right|>\left|z_{1}^{i}-z_{2}^{i}\right|\right\}\right)^{\frac{1}{2 d}} \leq c\left[\frac{\left(|t-s|^{\frac{2-\beta}{4}}+\|x-y\|^{\frac{2-\beta}{2}}\right)^{2}}{\left\|z_{1}-z_{2}\right\|^{2}} \wedge 1\right]^{\frac{p}{2 d}} .
$$

It remains to prove that

$$
\left\|H_{(1, \ldots, 2 d)}(Z, 1)\right\|_{0,2} \leq c_{T}\left(|t-s|^{\frac{2-\beta}{4}}+\|x-y\|^{\frac{2-\beta}{2}}\right)^{-d} .
$$

The proof of (3.4) is similar to that of [6, Proposition 5.11] by using the continuity of the Skorohod integral $\delta$ (see [11, Proposition 3.2.1] and [12, (1.11) and p. 131]) and Hölder's inequality for Malliavin norms (see [19, Proposition 1.10, p. 50]). Comparing with the estimate in [6, Proposition 5.11], we are able to remove the extra exponent $\eta$ because of the correct estimate on the inverse of the matrix $\gamma_{Z}$ in Proposition 3.4. 
Proof of Theorems 1.5. The proof is similar to that of [6, Theorem 1.2(b)]. We remark that the estimate in [6, Lemma 2.3] remains valid for $\tilde{\gamma}=\gamma=2-\beta$. Then by using Theorem 1.4, we follow along the same lines as in the proof of [6, Theorem 1.2(b)] with $d+\eta$ there replaced by $d$, to obtain the optimal lower bounds on hitting probabilities in terms of capacity.

\section{Proof of Theorems 1.1 and 1.2}

In this section, we establish the $L^{p}$-Hölder continuity of the solution and its Malliavin derivative. First, we recall some estimates on the Green kernel $S(t, x)$.

Lemma 4.1. There exist some $M_{0}, m_{0}>0$ such that for all $t>0, x, y \in \mathbb{R}^{k}$,

$$
|S(t, x)-S(t, y)| \leq M_{0}\|x-y\| t^{-\frac{k+1}{2}}\left(e^{-\frac{\|x\|^{2}}{m_{0} t}}+e^{-\frac{\|y\|^{2}}{m_{0} t}}\right) .
$$

Proof. This is a consequence of the mean-value theorem.

Lemma 4.2 ([1, Lemma 6.4]). There exist some $N_{0}>0$ such that for all $t>0, x, y \in \mathbb{R}^{k}$,

$$
\int_{\mathbb{R}^{k}}|S(t, x+z)-S(t, y+z)| d z \leq N_{0}\left(\frac{\|x-y\|}{t^{1 / 2}} \wedge 1\right) .
$$

Lemma 4.3. There exists a constant $C_{0}>0$ such that for all $t>0, x, y \in \mathbb{R}^{k}$,

$$
\int_{\mathbb{R}^{k}}\|z\|^{-\beta}|S(t, x+z)-S(t, y+z)| d z \leq C_{0} t^{-\beta / 2}\left(\frac{\|x-y\|}{t^{1 / 2}} \wedge 1\right) .
$$

Proof. First, by Lemma 4.1,

$$
\begin{aligned}
& \int_{\mathbb{R}^{k}}\|z\|^{-\beta}|S(t, x+z)-S(t, y+z)| d z \\
& \quad \leq M_{0}\|x-y\| t^{-\frac{k+1}{2}} \int_{\mathbb{R}^{k}}\|z\|^{-\beta}\left(e^{-\frac{\|x+z\|^{2}}{m_{0} t}}+e^{-\frac{\|y+z\|^{2}}{m_{0} t}}\right) d z \\
& \quad \leq 2 M_{0}\|x-y\| t^{-\frac{k+1}{2}} \sup _{x \in \mathbb{R}^{k}} \int_{\mathbb{R}^{k}}\|z\|^{-\beta} e^{-\frac{\|x+z\|^{2}}{m_{0} t}} d z \\
& \quad=2 M_{0}\|x-y\| t^{-\frac{k+1}{2}} \int_{\mathbb{R}^{k}}\|z\|^{-\beta} e^{-\frac{\|z\|^{2}}{m_{0} t}} d z \\
& \quad=C\|x-y\| t^{-\frac{\beta+1}{2}},
\end{aligned}
$$

where the first equality holds since the function $x \mapsto \int_{\mathbb{R}^{k}}\|z\|^{-\beta} e^{-\|x+z\|^{2} /\left(m_{0} t\right)} d z$ is a nonnegative definite function (its Fourier transform is a nonnegative function), which is therefore maximized at $x=0$.

On the other hand, using the same arguments as above,

$$
\begin{aligned}
\int_{\mathbb{R}^{k}} & \|z\|^{-\beta}|S(t, x+z)-S(t, y+z)| d z \\
& \leq \int_{\mathbb{R}^{k}}\|z\|^{-\beta}|S(t, x+z)+S(t, y+z)| d z \\
\leq & 2 \sup _{x \in \mathbb{R}^{k}} \int_{\mathbb{R}^{k}}\|z\|^{-\beta} S(t, x+z) d z \\
& =2 \int_{\mathbb{R}^{k}}\|z\|^{-\beta} S(t, z) d z=C t^{-\frac{\beta}{2}} .
\end{aligned}
$$

Therefore, (4.5) and (4.4) imply (4.3). 
Lemma 4.4. There exists a constant $C>0$ such that for all $s \geq 0, x, y \in \mathbb{R}^{k}$,

$$
\begin{aligned}
& \int_{0}^{s} d r \int_{\mathbb{R}^{k}} d z \int_{\mathbb{R}^{k}} d v\|z-v\|^{-\beta}|S(r, x-z)-S(r, y-z)||S(r, x-v)-S(r, y-v)| \\
& \quad \leq C\|x-y\|^{2-\beta} .
\end{aligned}
$$

Proof. By the change of variable $\left[z-v=v^{\prime}\right]$, the integral in (4.6) is equal to

$$
\begin{aligned}
& \int_{0}^{s} d r \int_{\mathbb{R}^{k}} d z|S(r, x-z)-S(r, y-z)| \\
& \quad \times \int_{\mathbb{R}^{k}} d v^{\prime}\left\|v^{\prime}\right\|^{-\beta}\left|S\left(r, x-z+v^{\prime}\right)-S\left(r, y-z+v^{\prime}\right)\right| .
\end{aligned}
$$

Applying Lemma 4.3 first and then Lemma 4.2, this is bounded above by

$$
\begin{aligned}
& C \int_{0}^{s} d r r^{-\beta / 2}\left(\frac{\|x-y\|}{r^{1 / 2}} \wedge 1\right) \int_{\mathbb{R}^{k}} d z|S(r, x-z)-S(r, y-z)| \\
& \quad \leq C \int_{0}^{s} d r r^{-\beta / 2}\left(\frac{\|x-y\|^{2}}{r} \wedge 1\right) \leq C \int_{0}^{\infty} d r r^{-\beta / 2}\left(\frac{\|x-y\|^{2}}{r} \wedge 1\right) \\
& \quad=C \int_{0}^{\|x-y\|^{2}} r^{-\beta / 2} d r+C\|x-y\|^{2} \int_{\|x-y\|^{2}}^{\infty} r^{-\beta / 2-1} d r \\
& \quad=C\|x-y\|^{2-\beta}+C\|x-y\|^{2+2 \times(1-1-\beta / 2)}=2 C\|x-y\|^{2-\beta} .
\end{aligned}
$$

Lemma 4.5. There exists a constant $C>0$ such that for all $t \geq 0, \delta \geq 0$ and $x \in \mathbb{R}^{k}$,

$$
\begin{aligned}
& \int_{0}^{t} d r \int_{\mathbb{R}^{k}} d z \int_{\mathbb{R}^{k}} d v\|z-v\|^{-\beta}|S(r+\delta, x-z)-S(r, x-z)||S(r+\delta, x-v)-S(r, x-v)| \\
& \quad \leq C \delta^{(2-\beta) / 2} .
\end{aligned}
$$

Proof. The proof follows the same lines as the proof of [9, Theorem 2]. We include the details here for reader's convenience. Denote $I_{1}$ the triple integral on the left-band side of (4.7). Since the two functions $z \mapsto\|z\|^{-\beta}$ and $z \mapsto S(r, z)$ are nonnegative definite, the convolution of these two functions is also nonnegative definite and hence maximized at 0 . Therefore, for all $x, v \in \mathbb{R}^{k}, \delta \geq 0$ and $r>0$,

$$
\begin{aligned}
& \int_{\mathbb{R}^{k}}\|z-v\|^{-\beta}|S(r+\delta, x-z)-S(r, x-z)| d z \\
& \quad \leq \int_{\mathbb{R}^{k}}\|z\|^{-\beta}(S(r+\delta, x-v-z)+S(r, x-v-z)) d z \\
& \leq \int_{\mathbb{R}^{k}}\|z\|^{-\beta}(S(r+\delta, z)+S(r, z)) d z \\
& =c\left((r+\delta)^{-\beta / 2}+r^{-\beta / 2}\right) \leq 2 c r^{-\beta / 2},
\end{aligned}
$$

where the equality follows from the fact $S(r, z)=r^{-k / 2} S(1, z / \sqrt{r})$. Therefore, for all $t \geq 0, \delta \geq 0$ and $x \in \mathbb{R}^{k}$,

$$
I_{1} \leq \int_{0}^{t} d r r^{-\beta / 2} \int_{\mathbb{R}^{k}} d v|S(r+\delta, x-v)-S(r, x-v)| .
$$

Since there are constants $c, C>0$ such that for all $r>0$ and $x \in \mathbb{R}^{k}, \frac{\partial S}{\partial r}(r, x) \leq \frac{C}{r} S(c r, x)$,

$$
\int_{\mathbb{R}^{k}} d v|S(r+\delta, x-v)-S(r, x-v)| \leq \int_{\mathbb{R}^{k}} d v \int_{r}^{r+\delta} d \rho \frac{C}{\rho} S(c \rho, x-v)
$$


Optimal lower bounds on hitting probabilities

$$
=C \int_{r}^{r+\delta} \frac{d \rho}{\rho}=\log (r+\delta)-\log r
$$

we see that

$$
\begin{aligned}
I_{1} & \leq C \int_{0}^{t} r^{-\beta / 2}(\log (r+\delta)-\log r) d r \\
& =\frac{2 C}{2-\beta}\left(\log (1+\delta / t) t^{(2-\beta) / 2}+\int_{0}^{t} r^{(2-\beta) / 2} \frac{\delta}{r(r+\delta)} d r\right) \\
& :=\frac{2 C}{2-\beta}\left(I_{3}+I_{4}\right) .
\end{aligned}
$$

For $I_{4}$, using the change of variables $s=r / \delta$,

$$
I_{4}=\delta^{(2-\beta) / 2} \int_{0}^{t / \delta} \frac{1}{s^{\beta / 2}(s+1)} d s \leq \delta^{(2-\beta) / 2} \int_{0}^{\infty} \frac{1}{s^{\beta / 2}(s+1)} d s=c \delta^{(2-\beta) / 2} .
$$

In order to estimate $I_{3}$, we use the fact that there exists $C>1$ such that for all $\mu>0$,

$$
0<\log (1+\mu) \leq C \mu^{(2-\beta) / 2} .
$$

Hence,

$$
I_{3}=\log (1+\delta / t) t^{(2-\beta) / 2} \leq C(\delta / t)^{(2-\beta) / 2} t^{(2-\beta) / 2}=C \delta^{(2-\beta) / 2} .
$$

Finally, (4.8)-(4.10) imply (4.7).

Remark 4.6. Regarding [9], we point out that in the case of the ordinary heat kernel, the argument for the estimate in [9, (2.26)] does not apply since the lower bound in [9, (1.7)] does not apply to the heat kernel. However, in the case of the heat kernel, the statement of $[9,(2.26)]$ (together with [9, (2.27) and (2.28)]) remains valid by the following calculation:

$$
\int_{\mathbb{R}^{k}}\|x\|^{-\beta} S(r, x) d x=\int_{\mathbb{R}^{k}} r^{-\beta / 2}\|z\|^{-\beta} S(1, z) d z=C r^{-\beta / 2} .
$$

Based on the above estimates on the Green kernel, we now prove Theorems 1.1 and 1.2 .

Proof of Theorem 1.1. By (1.14), it suffices to prove (1.7) when $t-s$ and $\|x-y\|$ are small. Without loss of generality, we assume that $t-s \leq 1 / 2$ and $\|x-y\| \leq 1 / 2$. Denote

$$
I_{i j}(t, x)=\int_{0}^{t} \int_{\mathbb{R}^{k}} S(t-\theta, x-\eta) \sigma_{i j}(u(\theta, \eta)) M^{j}(d \theta, d \eta) .
$$

From (1.12),

$$
\begin{aligned}
\left|u_{i}(t, x)-u_{i}(s, y)\right| \leq & \sum_{j=1}^{d}\left|I_{i j}(t, x)-I_{i j}(s, x)\right|+\sum_{j=1}^{d}\left|I_{i j}(s, x)-I_{i j}(s, y)\right| \\
& +\int_{s}^{t} \int_{\mathbb{R}^{k}} S(t-\theta, x-\eta)\left|b_{i}(u(\theta, \eta))\right| d \eta d \theta \\
& +\int_{0}^{s} \int_{\mathbb{R}^{k}}^{s}|S(t-\theta, x-\eta)-S(t-\theta, y-\eta)|\left|b_{i}(u(\theta, \eta))\right| d \eta d \theta \\
& +\int_{0}^{s} \int_{\mathbb{R}^{k}}|S(t-\theta, y-\eta)-S(s-\theta, y-\eta)|\left|b_{i}(u(\theta, \eta))\right| d \eta d \theta
\end{aligned}
$$


Optimal lower bounds on hitting probabilities

$$
:=I_{1}+I_{2}+I_{3}+I_{4}+I_{5} \text {. }
$$

By Burkholder's inequality, for any $p \geq 2$,

$$
\begin{aligned}
\mathrm{E}\left[\left|I_{1}\right|^{p}\right] \leq c \sum_{j=1}^{d} \mathrm{E}[\mid & \int_{s}^{t} d \theta \int_{\mathbb{R}^{k}} d z \int_{\mathbb{R}^{k}} d v \frac{1}{\|z-v\|^{\beta}} \\
& \left.\times S(t-\theta, x-z)\left|\sigma_{i j}(u(\theta, z))\right| S(t-\theta, x-v)\left|\sigma_{i j}(u(\theta, v))\right|^{\frac{p}{2}}\right] \\
+c \sum_{j=1}^{d} \mathrm{E}[ & {\left[\int_{0}^{s} d \theta \int_{\mathbb{R}^{k}} d z \int_{\mathbb{R}^{k}} d v \frac{1}{\|z-v\|^{\beta}}|S(t-\theta, x-z)-S(s-\theta, x-z)|\right.} \\
& \left.\times\left.\left|\sigma_{i j}(u(\theta, z))\right||S(t-\theta, x-v)-S(s-\theta, x-v)|\left|\sigma_{i j}(u(\theta, v))\right|\right|^{\frac{p}{2}}\right] .
\end{aligned}
$$

Using Minkowski inequality and the Cauchy-Schwartz inequality, (1.14) and the linear growth property of the functions $\sigma_{i j}$, this is bounded above by

$$
\begin{gathered}
c\left[\int_{s}^{t} d \theta \int_{\mathbb{R}^{k}} d z \int_{\mathbb{R}^{k}} d v \frac{1}{\|z-v\|^{\beta}} S(t-\theta, x-z) S(t-\theta, x-v)\right]^{\frac{p}{2}} \\
+c\left[\int_{0}^{s} d \theta \int_{\mathbb{R}^{k}} d z \int_{\mathbb{R}^{k}} d v \frac{1}{\|z-v\|^{\beta}}|S(t-\theta, x-z)-S(s-\theta, x-z)|\right. \\
\times|S(t-\theta, x-v)-S(s-\theta, x-v)|]^{\frac{p}{2}} .
\end{gathered}
$$

The first term above is equal to $c(t-s)^{(2-\beta) p / 4}$ by [6, (6.3)] and the second term above is bounded above by $c(t-s)^{(2-\beta) p / 4}$ by Lemma 4.5 . Hence for any $p \geq 2$,

$$
\mathrm{E}\left[\left|I_{1}\right|^{p}\right] \leq c(t-s)^{\frac{(2-\beta) p}{4}} .
$$

Similarly, applying Burkholder's inequality and taking the absolute value inside,

$$
\begin{gathered}
\mathrm{E}\left[\left|I_{2}\right|^{p}\right] \leq c \sum_{j=1}^{d} \mathrm{E}\left[|| \int_{0}^{s} d r \int_{\mathbb{R}^{k}} d z \int_{\mathbb{R}^{k}} d v\|z-v\|^{-\beta}|S(r, x-z)-S(r, y-z)|\left|\sigma_{i j}(u(s-r, z))\right|\right. \\
\left.\times\left.|S(r, x-v)-S(r, y-v)|\left|\sigma_{i j}(u(s-r, v))\right|\right|^{p / 2}\right] .
\end{gathered}
$$

By the Minkowski inequality with respect to the measure $\|z-v\|^{-\beta} \mid S(r, x-z)-S(r, y-$ $z)|| S(r, x-v)-S(r, y-v) \mid d r d v d z$, the Cauchy-Schwartz inequality, (1.14) and the linear growth property of the functions $\sigma_{i j}$, this is bounded above by

$$
\begin{aligned}
c \sup _{(t, x) \in[0, T] \times \mathbb{R}^{k}} \mathrm{E}\left[1+\|u(t, x)\|^{p}\right] \mid \int_{0}^{s} d r \int_{\mathbb{R}^{k}} d z \int_{\mathbb{R}^{k}} d v\|z-v\|^{-\beta} \\
\quad \times\left.|S(r, x-z)-S(r, y-z)||S(r, x-v)-S(r, y-v)|\right|^{p / 2} \\
\quad \leq c\|x-y\|^{\frac{(2-\beta) p}{2}}
\end{aligned}
$$

where the inequality follows from Lemma 4.4.

For the estimate of $I_{3}$, using the Minkowski inequality with respect to the measure $S(t-\theta, x-\eta) d \eta d \theta,(1.14)$ and the linear growth property of the functions $b_{i}$, we have

$$
\mathrm{E}\left[\left|I_{3}\right|^{p}\right] \leq c \sup _{(t, x) \in[0, T] \times \mathbb{R}^{k}} \mathrm{E}\left[1+\|u(t, x)\|^{p}\right]\left(\int_{s}^{t} \int_{\mathbb{R}^{k}} S(t-\theta, x-\eta) d \eta d \theta\right)^{p}
$$


Optimal lower bounds on hitting probabilities

$$
=c|t-s|^{p} .
$$

Moreover, using the Minkowski inequality with respect to the measure $\mid S(t-\theta, x-\eta)-$ $S(t-\theta, y-\eta) \mid d \eta d \theta,(1.14)$ and the linear growth property of the functions $b_{i}$,

$$
\begin{aligned}
\mathrm{E}\left[\left|I_{4}\right|^{p}\right] & \leq c\left(\int_{0}^{s} \int_{\mathbb{R}^{k}}|S(t-\theta, x-\eta)-S(t-\theta, y-\eta)| d \eta d \theta\right)^{p} \\
& \leq c\|x-y\|^{p},
\end{aligned}
$$

where the second inequality follows from [15, Lemme A2].

Similarly, by the Minkowski inequality with respect to the measure $\mid S(t-\theta, y-\eta)-$ $S(s-\theta, y-\eta) \mid d \eta d \theta,(1.14)$ and the linear growth property of the functions $b_{i}$,

$$
\begin{aligned}
\mathrm{E}\left[\left|I_{5}\right|^{p}\right] & \leq c\left(\int_{0}^{s} \int_{\mathbb{R}^{k}}|S(t-\theta, y-\eta)-S(s-\theta, y-\eta)| d \eta d \theta\right)^{p} \\
& \leq c|(t-s) \log (t-s)|^{p}
\end{aligned}
$$

where the second inequality follows from [15, Lemme A3].

Hence, (4.11)-(4.16) imply (1.7).

Proof of Theorem 1.2. The proof is similar to that of Theorem 1.1 by using Lemmas 4.4 and 4.5. From (2.3), we assume that $t-s \leq 1 / 2$ and $\|x-y\| \leq 1 / 2$.

We assume $m=1$ and fix $p \geq 2$. Let

$$
g_{t, x ; s, y}(r, *):=S(t-r, x-*) 1_{\{r<t\}}-S(s-r, y-*) 1_{\{r<s\}} .
$$

Using (2.1), we see that

$$
\left\|D\left(u_{i}(t, x)-u_{i}(s, y)\right)\right\|_{L^{p}\left(\Omega ; \mathscr{H}_{T}^{d}\right)}^{p} \leq c_{p}\left(A_{1,1}+A_{1,2}+A_{1,3}+A_{2,1}+A_{2,2}+A_{2,3}+A_{3,1}+A_{3,2}\right),
$$

where

$$
\begin{aligned}
& A_{1,1}=\mathrm{E}\left[\left(\int_{s}^{t} d r \sum_{j=1}^{d}\left\|S(t-r, x-*) \sigma_{i j}(u(r, *))\right\|_{\mathscr{H}}^{2}\right)^{p / 2}\right], \\
& A_{1,2}=\mathrm{E}\left[\left(\int_{0}^{s} d r \sum_{j=1}^{d}\left\|g_{t, x ; s, x}(r, *) \sigma_{i j}(u(r, *))\right\|_{\mathscr{H}}^{2}\right)^{p / 2}\right], \\
& A_{1,3}=\mathrm{E}\left[\left(\int_{0}^{s} d r \sum_{j=1}^{d}\left\|g_{s, x ; s, y}(r, *) \sigma_{i j}(u(r, *))\right\|_{\mathscr{H}}^{2}\right)^{p / 2}\right], \\
& A_{2,1}=\mathrm{E}\left[\left\|\int_{s}^{t} \int_{\mathbb{R}^{k}} S(t-\theta, x-\eta) \sum_{j=1}^{d} D\left(\sigma_{i j}(u(\theta, \eta))\right) M^{j}(d \theta, d \eta)\right\|_{\mathscr{H}_{T}^{d}}^{p}\right], \\
& A_{2,2}=\mathrm{E}\left[\left\|\int_{0}^{s} \int_{\mathbb{R}^{k}} g_{t, x ; s, x}(\theta, \eta) \sum_{j=1}^{d} D\left(\sigma_{i j}(u(\theta, \eta))\right) M^{j}(d \theta, d \eta)\right\|_{\mathscr{H}_{T}^{d}}^{p}\right], \\
& A_{2,3}=\mathrm{E}\left[\left\|\int_{0}^{s} \int_{\mathbb{R}^{k}} g_{s, x ; s, y}(\theta, \eta) \sum_{j=1}^{d} D\left(\sigma_{i j}(u(\theta, \eta))\right) M^{j}(d \theta, d \eta)\right\|_{\mathscr{H}_{T}^{d}}^{p}\right], \\
& A_{3,1}=\mathrm{E}\left[\left\|\int_{s}^{t} \int_{\mathbb{R}^{k}} S(t-\theta, x-\eta) D\left(b_{i}(u(\theta, \eta))\right) d \theta d \eta\right\|_{\mathscr{H}_{T}^{d}}^{p}\right], \\
& A_{3,2}=\mathrm{E}\left[\left\|\int_{0}^{s} \int_{\mathbb{R}^{k}}(S(t-\theta, x-\eta)-S(t-\theta, y-\eta)) D\left(b_{i}(u(\theta, \eta))\right) d \theta d \eta\right\|_{\mathscr{H}_{T}^{d}}^{p}\right],
\end{aligned}
$$


Optimal lower bounds on hitting probabilities

$$
A_{3,3}=\mathrm{E}\left[\left\|\int_{0}^{s} \int_{\mathbb{R}^{k}}(S(t-\theta, y-\eta)-S(s-\theta, y-\eta)) D\left(b_{i}(u(\theta, \eta))\right) d \theta d \eta\right\|_{\mathscr{H}_{T}^{d}}^{p}\right] .
$$

Using the Minkowski inequality, the Cauchy-Schwartz inequality, (1.14) and the linear growth property of the functions $\sigma_{i j}$, we have

$$
\begin{aligned}
A_{1,1} & \leq c \sup _{(t, x) \in[0, T] \times \mathbb{R}^{k}} \mathrm{E}\left[1+\|u(t, x)\|^{p}\right]\left(\int_{s}^{t} d r \int_{\mathbb{R}^{k}} \mu(d \xi)|\mathscr{F} S(t-r, x-*)(\xi)|^{2}\right)^{p / 2} \\
& =c\left(\int_{s}^{t}(t-r)^{-\beta / 2} d r\right)^{p / 2}=c^{\prime}(t-s)^{\frac{(2-\beta) p}{4}}
\end{aligned}
$$

where the first equality is due to $[6,(6.3)]$.

Similarly,

$$
\begin{aligned}
A_{1,2} \leq & c \sup _{(t, x) \in[0, T] \times \mathbb{R}^{k}} \mathrm{E}\left[1+\|u(t, x)\|^{p}\right]\left(\int_{0}^{s} d r \int_{\mathbb{R}^{k}} d z \int_{\mathbb{R}^{k}} d v\|z-v\|^{-\beta}\right. \\
& \times|S(t-r, x-z)-S(s-r, x-z)||S(t-r, x-v)-S(s-r, x-v)|)^{p / 2} \\
\leq & c(t-s)^{\frac{(2-\beta) p}{4}},
\end{aligned}
$$

where the last inequality follows from (1.14) and Lemma 4.5.

Moreover, by the Minkowski inequality, the Cauchy-Schwartz inequality, (1.14) and the linear growth property of $\sigma_{i j}$, we have

$$
\begin{aligned}
A_{1,3} \leq & c \sup _{(t, x) \in[0, T] \times \mathbb{R}^{k}} \mathrm{E}\left[1+\|u(t, x)\|^{p}\right]\left(\int_{0}^{s} d r \int_{\mathbb{R}^{k}} d z \int_{\mathbb{R}^{k}} d v\|z-v\|^{-\beta}\right. \\
& \times|S(s-r, x-z)-S(s-r, y-z)||S(s-r, x-v)-S(s-r, y-v)|)^{p / 2} \\
\leq & c\|x-y\|^{\frac{(2-\beta) p}{2}}
\end{aligned}
$$

where the last inequality follows from (1.14) and Lemma 4.4.

The estimate of $A_{2,1}$ is similar to that of $A_{1,1}$. Indeed, by Burkholder's inequality for Hilbert-space-valued martingales ([10, E.2. p. 212]),

$$
\begin{gathered}
A_{2,1} \leq c \sum_{j=1}^{d} \mathrm{E}\left[\left(\int_{s}^{t} d r \int_{\mathbb{R}^{k}} d z \int_{\mathbb{R}^{k}} d v\|z-v\|^{-\beta} S(t-r, x-z)\left\|D\left(\sigma_{i j}(u(\theta, z))\right)\right\|_{\mathscr{H}_{T}^{d}}\right.\right. \\
\left.\left.\times S(t-r, x-v)\left\|D\left(\sigma_{i j}(u(\theta, v))\right)\right\|_{\mathscr{H}_{T}^{d}}\right)^{p / 2}\right]
\end{gathered}
$$

By hypothesis P1, the Minkowski inequality, the Cauchy-Schwarz inequality and (2.3), this is bounded above by

$$
\begin{aligned}
& c \sum_{l=1}^{d} \sup _{(t, x) \in[0, T] \times \mathbb{R}^{k}} \mathrm{E}\left[\left\|D\left(u_{l}(t, x)\right)\right\|_{\mathscr{H}_{T}^{d}}^{p}\right] \\
& \quad \times\left(\int_{s}^{t} d r \int_{\mathbb{R}^{k}} d z \int_{\mathbb{R}^{k}} d v\|z-v\|^{-\beta} S(t-r, x-z) S(t-r, x-v)\right)^{p / 2} \\
& =c\left(\int_{s}^{t} d r \int_{\mathbb{R}^{k}} \mu(d \xi)|\mathscr{F} S(t-r, x-*)(\xi)|^{2}\right)^{p / 2} \\
& =c\left(\int_{s}^{t}(t-r)^{-\beta / 2} d r\right)^{p / 2}=c^{\prime}(t-s)^{\frac{(2-\beta) p}{4}},
\end{aligned}
$$


where the second equality is due to $[6,(6.3)]$.

Furthermore, by Burkholder's inequality for Hilbert-space-valued martingales ([10, E.2. p. 212]),

$$
\begin{aligned}
A_{2,2} \leq & c \sum_{j=1}^{d} \mathrm{E}\left[\left(\int_{0}^{s} d r \int_{\mathbb{R}^{k}} d z \int_{\mathbb{R}^{k}} d v\|z-v\|^{-\beta}|S(t-r, x-z)-S(s-r, x-z)|\right.\right. \\
& \left.\left.\times\left\|D\left(\sigma_{i j}(u(\theta, z))\right)\right\|_{\mathscr{H}_{T}^{d}}|S(t-r, x-v)-S(s-r, x-v)|\left\|D\left(\sigma_{i j}(u(\theta, v))\right)\right\|_{\mathscr{H}_{T}^{d}}\right)^{p / 2}\right] .
\end{aligned}
$$

Similar to the estimate of $A_{1,2}$, by hypothesis P1, the Minkowski inequality, the CauchySchwarz inequality and (2.3), this is bounded above by

$$
\begin{aligned}
& c \sum_{l=1}^{d} \sup _{(t, x) \in[0, T] \times \mathbb{R}^{k}} \mathrm{E}\left[\left\|D\left(u_{l}(t, x)\right)\right\|_{\mathscr{H}_{T}^{d}}^{p}\right]\left(\int_{0}^{s} d r \int_{\mathbb{R}^{k}} d z \int_{\mathbb{R}^{k}} d v\|z-v\|^{-\beta}\right. \\
& \quad \times|S(t-r, x-z)-S(s-r, x-z)||S(t-r, x-v)-S(s-r, x-v)|)^{p / 2} \\
& =c\left(\int_{0}^{s} d r \int_{\mathbb{R}^{k}} d z \int_{\mathbb{R}^{k}} d v\|z-v\|^{-\beta}|S(t-s+r, x-z)-S(r, x-z)|\right. \\
& \quad \times|S(t-s+r, x-v)-S(r, x-v)|)^{p / 2} \\
& \leq c(t-s)^{\frac{(2-\beta) p}{4}},
\end{aligned}
$$

where the last inequality follows from Lemma 4.5.

We move on to estimate $A_{2,3}$. By Burkholder's inequality for Hilbert-space-valued martingales ([10, E.2. p. 212]),

$$
\begin{aligned}
A_{2,3} \leq & c \sum_{j=1}^{d} \mathrm{E}\left[\left(\int_{0}^{s} d r \int_{\mathbb{R}^{k}} d z \int_{\mathbb{R}^{k}} d v\|z-v\|^{-\beta}|S(s-r, x-z)-S(s-r, y-z)|\right.\right. \\
& \left.\left.\times\left\|D\left(\sigma_{i j}(u(\theta, z))\right)\right\|_{\mathscr{H}_{T}^{d}}|S(s-r, x-v)-S(s-r, y-v)|\left\|D\left(\sigma_{i j}(u(\theta, v))\right)\right\|_{\mathscr{H}_{T}^{d}}\right)^{p / 2}\right] .
\end{aligned}
$$

Again, using hypothesis P1, the Minkowski inequality, the Cauchy-Schwarz inequality and (2.3), this is bounded above by

$$
\begin{aligned}
& c \sum_{l=1}^{d} \sup _{(t, x) \in[0, T] \times \mathbb{R}^{k}} \mathrm{E}\left[\left\|D\left(u_{l}(t, x)\right)\right\|_{\mathscr{H}_{T}^{d}}^{p}\right]\left(\int_{0}^{s} d r \int_{\mathbb{R}^{k}} d z \int_{\mathbb{R}^{k}} d v\|z-v\|^{-\beta}\right. \\
& \quad \times|S(s-r, x-z)-S(s-r, y-z)||S(s-r, x-v)-S(s-r, y-v)|)^{p / 2} \\
& =c\left(\int_{0}^{s} d r \int_{\mathbb{R}^{k}} d z \int_{\mathbb{R}^{k}} d v\|z-v\|^{-\beta}|S(r, x-z)-S(r, y-z)|\right. \\
& \quad \times|S(r, x-v)-S(r, y-v)|)^{p / 2} \\
& \leq c\|x-y\|^{\frac{(2-\beta) p}{2}},
\end{aligned}
$$

where the last inequality follows from Lemma 4.4.

We proceed to estimate $A_{3,1}, A_{3,2}$ and $A_{3,3}$. For $A_{3,1}$, by hypothesis P1, the Minkowski inequality and (2.3),

$$
A_{3,1} \leq c \sum_{l=1}^{d} \sup _{(t, x) \in[0, T] \times \mathbb{R}^{k}} \mathrm{E}\left[\left\|D\left(u_{l}(t, x)\right)\right\|_{\mathscr{H}_{T}^{d}}^{p}\right]\left(\int_{s}^{t} \int_{\mathbb{R}^{k}} S(t-\theta, x-\eta) d \theta d \eta\right)^{p}
$$


Optimal lower bounds on hitting probabilities

$$
=c(t-s)^{p} .
$$

Similar to the estimate of $I_{4}$ in the proof of Theorem 1.1, by hypothesis $\mathbf{P 1}$ and the Minkowski inequality,

$$
\begin{aligned}
A_{3,2} \leq c \sum_{l=1}^{d} & \sup _{(t, x) \in[0, T] \times \mathbb{R}^{k}} \mathrm{E}\left[\left\|D\left(u_{l}(t, x)\right)\right\|_{\mathscr{H}_{T}^{d}}^{p}\right] \\
& \times\left(\int_{0}^{s} \int_{\mathbb{R}^{k}}|S(t-\theta, x-\eta)-S(t-\theta, y-\eta)| d \eta d \theta\right)^{p} \\
& \leq c\|x-y\|^{p}
\end{aligned}
$$

where the second inequality follows from (2.3) and [15, Lemme A2].

Moreover, by hypothesis P1 and the Minkowski inequality,

$$
\begin{aligned}
& A_{3,3} \leq c \sum_{l=1}^{d} \sup _{(t, x) \in[0, T] \times \mathbb{R}^{k}} \mathrm{E}\left[\left\|D\left(u_{l}(t, x)\right)\right\|_{\mathscr{H}_{T}^{d}}^{p}\right] \\
& \times\left(\int_{0}^{s} \int_{\mathbb{R}^{k}}|S(t-\theta, y-\eta)-S(s-\theta, y-\eta)| d \eta d \theta\right)^{p} \\
& \leq c|(t-s) \log (t-s)|^{p}
\end{aligned}
$$

where the second inequality follows from (2.3) and [15, Lemme A3].

Therefore, (4.17)-(4.25) together prove (1.8) for $m=1$. The case $m>1$ follows along the same lines by induction using (2.3) and the stochastic partial differential equations satisfied by the iterated derivatives (cf. [13, Proposition 6.1]).

\section{Proof of Theorem 1.3}

We first state an elementary fact that will be used several times later on.

Lemma 5.1. Fix $\gamma \in] 0,1[$ and $\mu>0$.

(a) The function $x \mapsto(x+\mu)^{\gamma}-x^{\gamma}$ is nonincreasing on $[0, \infty[$ and the function $x \mapsto$ $x^{\gamma}-(x-\mu)^{\gamma}$ is nonincreasing on $[\mu, \infty[$.

(b) $(1+x)^{\gamma}-1 \leq \gamma x$ for all $x \geq 0$.

We recall from [6, p. 148] an estimate on the Malliavin derivative of the solution.

Lemma 5.2. For all $q \geq 1,0<\epsilon \leq s \leq T$ and $s-\epsilon \leq \rho \leq T$, there exists $C>0$ such that for all $i \in\{1, \ldots, d\}$,

$$
\begin{aligned}
\sup _{x \in \mathbb{R}^{k}} \mathrm{E}\left[\left\|D_{\cdot, *}\left(u_{i}(\rho, x)\right)\right\|_{\mathscr{H}_{s-\epsilon, s}^{d}}^{2 q}\right] & \leq C\left(\int_{s-\epsilon}^{s \wedge \rho} d \theta \int_{\mathbb{R}^{k}} \mu(d \xi)|\mathscr{F} S(\rho-\theta, *)(\xi)|^{2}\right)^{q} \\
& \leq C \epsilon^{\frac{(2-\beta) q}{2}} .
\end{aligned}
$$

Note that (5.1) is exactly the estimate between (6.2) and (6.3) in [6, p. 148] and (5.2) follows from the calculation below [6, (6.3)]. We give the proof of (5.1) in the appendix for reader's convenience.

We next give an estimate on $a_{i}(l, r, t, x)$, which is a refinement of [6, Lemma 6.2].

Lemma 5.3. Assume P1. Fix $T>0, c_{0}>1$ and $0<\gamma_{0}<1$. For any $q \geq 1$, there exists a constant $c=c\left(c_{0}, \gamma_{0}, q, T\right)>0$ such that for every $0<\epsilon \leq s \leq t \leq T$ with $t-s>c_{0} \epsilon^{\gamma_{0}}$ and $x \in \mathbb{R}^{k}$,

$$
W:=\mathrm{E}\left[\sup _{\xi \in \mathbb{R}^{d}:\|\xi\| \leq 1}\left(\int_{s-\epsilon}^{s} d r \sum_{l=1}^{d}\left\|\sum_{i=1}^{d} a_{i}(l, r, t, x) \xi_{i}\right\|_{\mathscr{H}}^{2}\right)^{q}\right]
$$


Optimal lower bounds on hitting probabilities

$$
\leq c \epsilon^{\min \left\{\frac{2-\beta}{2}\left(1+\gamma_{0}\right), 1-\frac{\beta \gamma_{0}}{2}\right\} q},
$$

where $a_{i}(l, r, t, x)$ is defined in (2.2).

Proof. We adopt the same notation as in the proof of [6, Lemma 6.2]. Use (2.2) and the Cauchy-Schwartz inequality to get

$$
W \leq c\left(\left(\sum_{i, j=1}^{d} \mathrm{E}\left[\left(\int_{s-\epsilon}^{s} d r\left\|W_{1}\right\|_{\mathscr{H} C^{d}}^{2}\right)^{q}\right]\right)+\left(\sum_{i=1}^{d} \mathrm{E}\left[\left(\int_{s-\epsilon}^{s} d r\left\|W_{2}\right\|_{\mathscr{H} d}^{2}\right)^{q}\right]\right)\right),
$$

where

$$
\begin{aligned}
& W_{1}=\int_{r}^{t} \int_{\mathbb{R}^{k}} S(t-\theta, x-\eta) D_{r}\left(\sigma_{i j}(u(\theta, \eta))\right) M^{j}(d \theta, d \eta), \\
& W_{2}=\int_{r}^{t} d \theta \int_{\mathbb{R}^{k}} d \eta S(t-\theta, x-\eta) D_{r}\left(b_{i}(u(\theta, \eta))\right) .
\end{aligned}
$$

Then

$$
\mathrm{E}\left[\left(\int_{s-\epsilon}^{s} d r\left\|W_{1}\right\|_{\mathscr{H}^{d}}^{2}\right)^{q}\right]=\mathrm{E}\left[\left\|W_{1}\right\|_{\mathscr{H}_{s-\epsilon, s}^{d}}^{2 q}\right] .
$$

Using hypothesis P1, Burkholder's inequality for Hilbert-space-valued martingales ([10, E.2. p. 212]) ensures that this is

$$
\begin{aligned}
\leq & c \sum_{l=1}^{d} \mathrm{E}\left[\left(\int_{s-\epsilon}^{t} d r \int_{\mathbb{R}^{k}} d z \int_{\mathbb{R}^{k}} d v\|z-v\|^{-\beta} S(t-r, x-z)\left\|D_{\cdot, *}\left(u_{l}(r, z)\right)\right\|_{\mathscr{H}_{s-\epsilon, s}^{d}}\right.\right. \\
& \left.\left.\times S(t-r, x-v)\left\|D_{\cdot, *}\left(u_{l}(r, v)\right)\right\|_{\mathscr{H}_{s-\epsilon, s}^{d}}\right)^{q}\right] \\
\leq I_{1,1}+I_{1,2}+ & I_{1,3},
\end{aligned}
$$

where for $i=1,2,3$,

$$
\begin{aligned}
I_{1, i}:=c \sum_{l=1}^{d} \mathrm{E}\left[\left(\int_{a_{i}}^{b_{i}} d r \int_{\mathbb{R}^{k}} d z \int_{\mathbb{R}^{k}} d v\|z-v\|^{-\beta} S(t-r, x-z)\left\|D_{\cdot, *}\left(u_{l}(r, z)\right)\right\|_{\mathscr{H}_{s-\epsilon, s}^{d}}\right.\right. \\
\left.\left.\times S(t-r, x-v)\left\|D_{\cdot, *}\left(u_{l}(r, v)\right)\right\|_{\mathscr{H}_{s-\epsilon, s}^{d}}\right)^{q}\right]
\end{aligned}
$$

and

$$
a_{1}=s-\epsilon, b_{1}=s, a_{2}=s, b_{2}=s+c_{0} \epsilon^{\gamma_{0}}, a_{3}=s+c_{0} \epsilon^{\gamma_{0}}, b_{3}=t .
$$

We now estimate $I_{1, i}$. Applying Hölder's inequality and the Cauchy-Schwartz inequality,

$$
\begin{aligned}
I_{1, i} \leq & c \sum_{l=1}^{d}\left(\int_{a_{i}}^{b_{i}} d r \int_{\mathbb{R}^{k}} \mu(d \xi)|\mathscr{F} S(t-r, x-*)(\xi)|^{2}\right)^{q-1} \\
& \times \int_{a_{i}}^{b_{i}} d r \int_{\mathbb{R}^{k}} \mu(d \xi)|\mathscr{F} S(t-r, x-*)(\xi)|^{2} \sup _{\eta \in \mathbb{R}^{k}} \mathrm{E}\left[\left\|D_{\cdot, *}\left(u_{l}(r, \eta)\right)\right\|_{\mathscr{H}_{s-\epsilon, s}^{d}}^{2 q}\right] .
\end{aligned}
$$

In the case $i=1$, we find that, by (5.2) and [6, (6.3)],

$$
I_{1,1} \leq c \epsilon^{\frac{(2-\beta) q}{2}}\left(\int_{s-\epsilon}^{s} d r \int_{\mathbb{R}^{k}} \mu(d \xi)|\mathscr{F} S(t-r, x-*)(\xi)|^{2}\right)^{q}
$$


Optimal lower bounds on hitting probabilities

$$
\begin{aligned}
& =c \epsilon^{\frac{(2-\beta) q}{2}}\left((t-s+\epsilon)^{(2-\beta) / 2}-(t-s)^{(2-\beta) / 2}\right)^{q} \\
& \leq c \epsilon^{\frac{(2-\beta) q}{2}}\left(\left(c_{0} \epsilon^{\gamma_{0}}+\epsilon\right)^{(2-\beta) / 2}-\left(c_{0} \epsilon^{\gamma_{0}}\right)^{(2-\beta) / 2}\right)^{q} \\
& =c \epsilon^{\frac{(2-\beta)\left(1+\gamma_{0}\right) q}{2}}\left(\left(1+c_{0}^{-1} \epsilon^{1-\gamma_{0}}\right)^{(2-\beta) / 2}-1\right)^{q} \\
& \leq c \epsilon^{\frac{(2-\beta)\left(1+\gamma_{0}\right) q}{2}}+\left(1-\gamma_{0}\right) q
\end{aligned}
$$

where the second inequality follows from Lemma 5.1(a) because $t-s>c_{0} \epsilon^{\gamma_{0}}$, and the third inequality is due to Lemma 5.1(b).

Similarly, by (5.7),

$$
\begin{aligned}
I_{1,2} & \leq c \epsilon^{\frac{(2-\beta) q}{2}}\left((t-s)^{(2-\beta) / 2}-\left(t-s-c_{0} \epsilon^{\gamma_{0}}\right)^{(2-\beta) / 2}\right)^{q} \\
& \leq c \epsilon^{\frac{(2-\beta) q}{2}}\left(c_{0} \epsilon^{\gamma_{0}}\right)^{(2-\beta) q / 2}=c^{\prime} \epsilon^{\frac{2-\beta}{2}\left(1+\gamma_{0}\right) q},
\end{aligned}
$$

where the second inequality holds by Lemma 5.1(a) since $t-s>c_{0} \epsilon^{\gamma_{0}}$.

Moreover, by (5.7), using [6, (6.3)] and (5.1),

$$
\begin{aligned}
I_{1,3} & \leq c\left(t-s-c_{0} \epsilon^{\gamma_{0}}\right)^{\frac{(2-\beta)(q-1)}{2}} \int_{s+c_{0} \epsilon^{\gamma_{0}}}^{t} d r \int_{\mathbb{R}^{k}} \mu(d \xi)|\mathscr{F} S(t-r, x-*)(\xi)|^{2} \\
& \times\left(\int_{s-\epsilon}^{s} d \theta \int_{\mathbb{R}^{k}} \mu(d \xi)|\mathscr{F} S(r-\theta, *)(\xi)|^{2}\right)^{q} \\
& =c\left(t-s-c_{0} \epsilon^{\gamma_{0}}\right)^{\frac{(2-\beta)(q-1)}{2}} \int_{s+c_{0} \epsilon^{\gamma_{0}}}^{t} d r \int_{\mathbb{R}^{k}} \mu(d \xi)|\mathscr{F} S(t-r, x-*)(\xi)|^{2} \\
& \leq c\left(t-s-c_{0} \epsilon^{\gamma_{0}}\right)^{\frac{(2-\beta) q}{2}}\left(\left(c_{0} \epsilon^{\gamma_{0}}+\epsilon\right)^{\frac{2-\beta}{2}}-\left(c_{0} \epsilon^{\gamma_{0}}\right)^{\frac{2-\beta}{2}}\right)^{q} \\
& \leq c \epsilon^{\frac{2-\beta}{2} \gamma_{0} q}\left(\left(1+c_{0}^{-1} \epsilon^{1-\gamma_{0}}\right)^{\frac{2-\beta}{2}}-1\right)^{q} \\
& \leq c \epsilon^{\left(\frac{2-\beta}{2} \gamma_{0}+1-\gamma_{0}\right) q}=c \epsilon^{\left(1-\beta \gamma_{0} / 2\right) q},
\end{aligned}
$$

where in the equality we use [6, Lemma 6.1], the second inequality follows from Lemma 5.1(a) since $r-s \geq c_{0} \epsilon^{\gamma_{0}}$ for all $r \in\left[s+c_{0} \epsilon^{\gamma_{0}}, t\right]$, in the third inequality we bound $t-s-c_{0} \epsilon^{\gamma_{0}}$ by $T$, and in the fourth inequality we use Lemma 5.1(b).

We proceed to estimate the second term in (5.4). First, by hypothesis P1 and the Cauchy-Schwartz inequality,

$$
\begin{aligned}
\int_{s-\epsilon}^{s} d r\left\|W_{2}\right\|_{\mathscr{H}^{d}}^{2} & \leq c \sum_{l=1}^{d} \int_{s-\epsilon}^{s} d r\left(\int_{r}^{t} d \rho \int_{\mathbb{R}^{k}} d \xi S(t-\rho, x-\xi)\left\|D_{r}\left(u_{l}(\rho, \xi)\right)\right\|_{\mathscr{H}^{d}}\right)^{2} \\
& \leq c \sum_{l=1}^{d}(t-s+\epsilon) \int_{s-\epsilon}^{s} d r \int_{r}^{t} d \rho \int_{\mathbb{R}^{k}} d \xi S(t-\rho, x-\xi)\left\|D_{r}\left(u_{l}(\rho, \xi)\right)\right\|_{\mathscr{H}^{d}}^{2} \\
& \leq c \sum_{l=1}^{d} \int_{s-\epsilon}^{t} d \rho \int_{\mathbb{R}^{k}} d \xi S(t-\rho, x-\xi)\left\|D_{\cdot, *}\left(u_{l}(\rho, \xi)\right)\right\|_{\mathscr{H}_{s-\epsilon, s}^{d}}^{2} .
\end{aligned}
$$

Therefore,

$$
\mathrm{E}\left[\left(\int_{s-\epsilon}^{s} d r\left\|W_{2}\right\|_{\mathscr{H}^{d}}^{2}\right)^{q}\right] \leq c\left(I_{2,1}+I_{2,2}+I_{2,3}\right),
$$

where for $i=1,2,3$,

$$
I_{2, i}=\sum_{l=1}^{d} \mathrm{E}\left[\left(\int_{a_{i}}^{b_{i}} d \rho \int_{\mathbb{R}^{k}} d \xi S(t-\rho, x-\xi)\left\|D_{\cdot, *}\left(u_{l}(\rho, \xi)\right)\right\|_{\mathscr{H}_{s-\epsilon, s}^{d}}^{2}\right)^{q}\right]
$$


and $a_{i}, b_{i}$ are defined in (5.6). By Hölder's inequality,

$$
\begin{aligned}
I_{2, i} \leq c & \sum_{l=1}^{d}\left(\int_{a_{i}}^{b_{i}} d \rho \int_{\mathbb{R}^{k}} d \xi S(t-\rho, x-\xi)\right)^{q-1} \\
& \times \int_{a_{i}}^{b_{i}} d \rho \int_{\mathbb{R}^{k}} d \xi S(t-\rho, x-\xi) \mathrm{E}\left[\left\|D_{\cdot, *}\left(u_{l}(\rho, \xi)\right)\right\|_{\mathscr{H}_{s-\epsilon, s}^{d}}^{2 q}\right] .
\end{aligned}
$$

In the case $i=1$, by (5.2),

$$
I_{2,1} \leq c \epsilon^{\frac{(2-\beta) q}{2}}\left(\int_{s-\epsilon}^{s} d \rho \int_{\mathbb{R}^{k}} d \xi S(t-\rho, x-\xi)\right)^{q}=c \epsilon^{(2-\beta / 2) q} .
$$

Similarly, by (5.11),

$$
\begin{aligned}
I_{2,2} & \leq c \epsilon^{\frac{(2-\beta) q}{2}}\left(\int_{s}^{s+c_{0} \epsilon^{\gamma_{0}}} d \rho \int_{\mathbb{R}^{k}} d \xi S(t-\rho, x-\xi)\right)^{q} \\
& =c \epsilon^{\gamma_{0} q} \epsilon^{\frac{(2-\beta) q}{2}}=c \epsilon^{\left(1+\gamma_{0}-\beta / 2\right) q} .
\end{aligned}
$$

It remains to estimate $I_{2,3}$. By (5.11), (5.1) and [6, (6.3)],

$$
\begin{aligned}
I_{2,3} & \leq c\left(t-s-c_{0} \epsilon^{\gamma_{0}}\right)^{q-1} \int_{s+c_{0} \epsilon^{\gamma_{0}}}^{t} d \rho\left(\int_{s-\epsilon}^{s \wedge \rho} d \theta \int_{\mathbb{R}^{k}} \mu(d \xi)|\mathscr{F} S(\rho-\theta, *)(\xi)|^{2}\right)^{q} \\
& =c\left(t-s-c_{0} \epsilon^{\gamma_{0}}\right)^{q-1} \int_{s+c_{0} \epsilon^{\gamma_{0}}}^{t} d \rho\left((\rho-s+\epsilon)^{\frac{2-\beta}{2}}-(\rho-s)^{\frac{2-\beta}{2}}\right)^{q} \\
& \leq c\left(t-s-c_{0} \epsilon^{\gamma_{0}}\right)^{q}\left(\left(c_{0} \epsilon^{\gamma_{0}}+\epsilon\right)^{\frac{2-\beta}{2}}-\left(c_{0} \epsilon^{\gamma_{0}}\right)^{\frac{2-\beta}{2}}\right)^{q} \\
& \leq c \epsilon^{\frac{2-\beta}{2} \gamma_{0} q}\left(\left(1+c_{0}^{-1} \epsilon^{1-\gamma_{0}}\right)^{\frac{2-\beta}{2}}-1\right)^{q} \\
& \leq c \epsilon^{\left(\frac{2-\beta}{2} \gamma_{0}+1-\gamma_{0}\right) q}=c \epsilon^{\left(1-\beta \gamma_{0} / 2\right) q}
\end{aligned}
$$

where in the second inequality we use Lemma 5.1(a) since $\rho-s \geq c_{0} \epsilon^{\gamma_{0}}$ for all $\rho \in$ $\left[s+c_{0} \epsilon^{\gamma_{0}}, t\right]$, in the third inequality we bound $t-s-c_{0} \epsilon^{\gamma_{0}}$ by $T$, and in the fourth inequality we use Lemma 5.1(b).

Finally, we combine the estimates in (5.8)-(5.14) to obtain (5.3).

We now prove Theorem 1.3.

Proof of Theorem 1.3. The proof of this theorem follows lines similar to those of [6, Proposition 5.6].

Case 1. Assume $t-s>0$ and $\|x-y\|^{2} \leq t-s$. Fix $\left.\epsilon \in\right] 0, \delta(t-s)[$, where $0<\delta<1$ is fixed; its specific value will be decided on later (see the line above (5.52)). For $\xi=(\lambda, \mu) \in \mathbb{R}^{2 d}$ with $\|\xi\|^{2}=\|\lambda\|^{2}+\|\mu\|^{2}=1$, we write

$$
\xi^{T} \gamma_{Z} \xi \geq J_{1}+J_{2},
$$

where

$$
\begin{aligned}
J_{1} & :=\int_{s-\epsilon}^{s} d r \sum_{l=1}^{d}\left\|\sum_{i=1}^{d}\left(\lambda_{i}-\mu_{i}\right)\left[S(s-r, y-*) \sigma_{i l}(u(r, *))+a_{i}(l, r, s, y)\right]+W\right\|_{\mathscr{H}}^{2}, \\
J_{2} & :=\int_{t-\epsilon}^{t} d r \sum_{l=1}^{d}\|W\|_{\mathscr{H}}^{2},
\end{aligned}
$$


where

$$
W:=\sum_{i=1}^{d}\left[\mu_{i} S(t-r, x-*) \sigma_{i l}(u(r, *))+\mu_{i} a_{i}(l, r, t, x)\right],
$$

and $a_{i}(l, r, t, x)$ is defined in (2.2).

We use the inequality

$$
\|a+b\|_{\mathscr{H}}^{2} \geq \frac{2}{3}\|a\|_{\mathscr{H}}^{2}-2\|b\|_{\mathscr{H}}^{2},
$$

subtract and add a "local" term to find that $J_{2} \geq \frac{2}{3} J_{2}^{(1)}-4\left(J_{2}^{(2)}+J_{2}^{(3)}\right)$, where

$$
\begin{aligned}
J_{2}^{(1)}= & \sum_{l=1}^{d} \int_{t-\epsilon}^{t} d r \int_{\mathbb{R}^{k}} d v \int_{\mathbb{R}^{k}} d z\|v-z\|^{-\beta} \\
& \times S(t-r, x-v) S(t-r, x-z)\left(\mu^{T} \cdot \sigma(u(r, x))\right)_{l}^{2}, \\
J_{2}^{(2)}= & \sum_{l=1}^{d} \int_{t-\epsilon}^{t} d r \int_{\mathbb{R}^{k}} d v \int_{\mathbb{R}^{k}} d z\|v-z\|^{-\beta} S(t-r, x-v) S(t-r, x-z) \\
& \times\left(\mu^{T} \cdot[\sigma(u(r, v))-\sigma(u(r, x))]\right)_{l}\left(\mu^{T} \cdot[\sigma(u(r, z))-\sigma(u(r, x))]\right)_{l}, \\
J_{2}^{(3)}= & \int_{t-\epsilon}^{t} d r \sum_{l=1}^{d}\left\|\sum_{i=1}^{d} a_{i}(l, r, t, x) \mu_{i}\right\|_{\mathscr{H}}^{2} .
\end{aligned}
$$

Now, hypothesis $\mathbf{P 2}$ and [6, Lemma 6.1] together imply that

$$
J_{2}^{(1)} \geq c\|\mu\| \epsilon^{\frac{2-\beta}{2}} .
$$

Similar to the calculation in [6, (4.4)], we can replace the exponent $\gamma$ there by $2-\beta$ by using our Theorem 1.1 instead of their (2.6) to obtain that, for any $q \geq 1$,

$$
\mathrm{E}\left[\sup _{\|\xi\|=1}\left|J_{2}^{(2)}\right|^{q}\right] \leq c \epsilon^{(2-\beta) q} .
$$

Moreover, applying [6, Lemma 6.2] with $a=1$ and $s=t$,

$$
\mathrm{E}\left[\sup _{\|\xi\|=1}\left|J_{2}^{(3)}\right|^{q}\right] \leq c \epsilon^{(2-\beta) q} .
$$

We will bound $J_{1}$ in two different subcases.

Subcase A: $\epsilon<\delta(t-s)^{1 / \gamma_{0}}$ where $\left.\gamma_{0} \in\right] \frac{1}{2}, 1[$. We use (5.18) again and we subtract and add a "local" term to see that

$$
J_{1} \geq \frac{2}{3} J_{1}^{(1)}-8\left(J_{1}^{(2)}+J_{1}^{(3)}+J_{1}^{(4)}+J_{1}^{(5)}\right),
$$

where

$$
\begin{aligned}
J_{1}^{(1)}= & \sum_{l=1}^{d} \int_{s-\epsilon}^{s} d r\left((\lambda-\mu)^{T} \cdot \sigma(u(r, y))\right)_{l}^{2} \int_{\mathbb{R}^{k}} d \xi\|\xi\|^{\beta-k}|\mathscr{F} S(s-r, y-*)(\xi)|^{2}, \\
J_{1}^{(2)}= & \sum_{l=1}^{d} \int_{s-\epsilon}^{s} d r \int_{\mathbb{R}^{k}} d v \int_{\mathbb{R}^{k}} d z\|v-z\|^{-\beta} S(s-r, y-v) S(s-r, y-z) \\
& \quad \times\left((\lambda-\mu)^{T} \cdot[\sigma(u(r, v))-\sigma(u(r, y))]\right)_{l}\left((\lambda-\mu)^{T} \cdot[\sigma(u(r, z))-\sigma(u(r, y))]\right)_{l},
\end{aligned}
$$




$$
\begin{aligned}
& J_{1}^{(3)}=\int_{s-\epsilon}^{s} d r \sum_{l=1}^{d}\left\|\sum_{i=1}^{d} \mu_{i} S(t-r, x-*) \sigma_{i l}(u(r, *))\right\|_{\mathscr{H}}^{2}, \\
& J_{1}^{(4)}=\int_{s-\epsilon}^{s} d r \sum_{l=1}^{d}\left\|\sum_{i=1}^{d}\left(\lambda_{i}-\mu_{i}\right) a_{i}(l, r, s, y)\right\|_{\mathscr{H}}^{2}, \\
& J_{1}^{(5)}=\int_{s-\epsilon}^{s} d r \sum_{l=1}^{d}\left\|\sum_{i=1}^{d} \mu_{i} a_{i}(l, r, t, x)\right\|_{\mathscr{H}}^{2} .
\end{aligned}
$$

Hypothesis $\mathbf{P 2}$ and [6, Lemma 6.1] together imply that

$$
J_{1}^{(1)} \geq c\|\lambda-\mu\| \epsilon^{\frac{2-\beta}{2}} .
$$

Similar to the term $J_{2}^{(2)}$ and (5.20), we obtain that, for any $q \geq 1$,

$$
\mathrm{E}\left[\sup _{\|\xi\|=1}\left|J_{1}^{(2)}\right|^{q}\right] \leq c \epsilon^{(2-\beta) q} .
$$

Using hypothesis $\mathbf{P 1}$ and [6, lemma 6.1],

$$
\begin{aligned}
J_{1}^{(3)} & \leq c \int_{s-\epsilon}^{s} d r \int_{\mathbb{R}^{k}} d \xi\|\xi\|^{\beta-k}|\mathscr{F} S(t-r, x-*)(\xi)|^{2} \\
& =c\left((t-s+\epsilon)^{\frac{2-\beta}{2}}-(t-s)^{\frac{2-\beta}{2}}\right) \\
& \leq c\left(\left(\delta^{-\gamma_{0}} \epsilon^{\gamma_{0}}+\epsilon\right)^{\frac{2-\beta}{2}}-\left(\delta^{-\gamma_{0}} \epsilon^{\gamma_{0}}\right)^{\frac{2-\beta}{2}}\right) \\
& =c \epsilon^{\frac{2-\beta}{2} \gamma_{0}}\left(\left(1+\delta^{\gamma_{0}} \epsilon^{1-\gamma_{0}}\right)^{\frac{2-\beta}{2}}-1\right) \leq c^{\prime} \epsilon^{1-\beta \gamma_{0} / 2},
\end{aligned}
$$

where the second inequality holds by Lemma 5.1(a) because $t-s>\delta^{-\gamma_{0}} \epsilon^{\gamma_{0}}$, and the last inequality holds by Lemma 5.1(b).

Similar to the term $J_{2}^{(3)}$ and (5.21), we see that, for any $q \geq 1$,

$$
\mathrm{E}\left[\sup _{\|\xi\|=1}\left|J_{1}^{(4)}\right|^{q}\right] \leq c \epsilon^{(2-\beta) q} .
$$

To estimate $J_{1}^{(5)}$, since we are under the assumption $t-s>\delta^{-\gamma_{0}} \epsilon^{\gamma_{0}}$, by Lemma 5.3, for any $q \geq 1$,

$$
\mathrm{E}\left[\sup _{\|\xi\|=1}\left|J_{1}^{(5)}\right|^{q}\right] \leq c \epsilon^{\min \left\{\frac{2-\beta}{2}\left(1+\gamma_{0}\right), 1-\frac{\beta \gamma_{0}}{2}\right\} q}
$$

From (5.19)-(5.26), we conclude that in the subcase $\epsilon<\delta(t-s)^{1 / \gamma_{0}}$,

$$
\inf _{\|\xi\|=1} \xi^{T} \gamma_{Z} \xi \geq c \epsilon^{\frac{2-\beta}{2}}-Z_{\epsilon}^{1},
$$

where $Z_{\epsilon}^{1}:=\sup _{\|\xi\|=1} 8\left(J_{2}^{(2)}+J_{2}^{(3)}+J_{1}^{(2)}+J_{1}^{(3)}+J_{1}^{(4)}+J_{1}^{(5)}\right)$ satisfies that, for any $q \geq 1$,

$$
\mathrm{E}\left[\sup _{\|\xi\|=1}\left|Z_{\epsilon}^{1}\right|^{q}\right] \leq c \epsilon^{\min \left\{\frac{2-\beta}{2}\left(1+\gamma_{0}\right), 1-\frac{\beta \gamma_{0}}{2}\right\} q} .
$$

Subcase $B: \delta(t-s)^{1 / \gamma_{0}} \leq \epsilon<\delta(t-s)$. In this subcase, we give a different estimate on $J_{1}$. Apply inequality (5.18) and subtract and add a "local" term, to find that

$$
J_{1} \geq \frac{2}{3} A_{1}-8\left(A_{2}+A_{3}+A_{4}+A_{5}\right),
$$


where

$$
\begin{aligned}
A_{1}=\sum_{l=1}^{d} \int_{s-\epsilon}^{s} d r \| S(s-r, y-*)\left((\lambda-\mu)^{T} \cdot \sigma(u(r, y))\right)_{l} \\
\quad+S(t-r, x-*)\left(\mu^{T} \cdot \sigma(u(r, x))\right)_{l} \|_{\mathscr{H}}^{2}, \\
A_{2}=\sum_{l=1}^{d} \int_{s-\epsilon}^{s} d r\left\|S(s-r, y-*)\left((\lambda-\mu)^{T} \cdot[\sigma(u(r, *))-\sigma(u(r, y))]\right)_{l}\right\|_{\mathscr{H}}^{2}, \\
A_{3}=\sum_{l=1}^{d} \int_{s-\epsilon}^{s} d r\left\|S(t-r, x-*)\left(\mu^{T} \cdot[\sigma(u(r, *))-\sigma(u(r, x))]\right)_{l}\right\|_{\mathscr{H}}^{2}, \\
A_{4}=\sum_{l=1}^{d} \int_{s-\epsilon}^{s} d r\left\|\sum_{i=1}^{d}\left(\lambda_{i}-\mu_{i}\right) a_{i}(l, r, s, y)\right\|_{\mathscr{H}}^{2}, \\
A_{5}=\sum_{l=1}^{d} \int_{s-\epsilon}^{s} d r\left\|\sum_{i=1}^{d} \mu_{i} a_{i}(l, r, t, x)\right\|_{\mathscr{H}}^{2} \cdot
\end{aligned}
$$
where

Using the inequality $\|a+b\|_{\mathscr{H}}^{2} \geq\|a\|^{2}+\|b\|^{2}-2\left|\langle a, b\rangle_{\mathscr{H}}\right|$, we see that $A_{1} \geq \tilde{A}_{1}+\tilde{A}_{2}-2 \tilde{B}_{4}$,

$$
\begin{aligned}
& \tilde{A}_{1}=\sum_{l=1}^{d} \int_{s-\epsilon}^{s} d r\left\|S(s-r, y-*)\left((\lambda-\mu)^{T} \cdot \sigma(u(r, y))\right)_{l}\right\|_{\mathscr{H}}^{2}, \\
& \tilde{A}_{2}=\sum_{l=1}^{d} \int_{s-\epsilon}^{s} d r\left\|S(t-r, x-*)\left(\mu^{T} \cdot \sigma(u(r, x))\right)_{l}\right\|_{\mathscr{H}}^{2}, \\
& \tilde{B}_{4}=\sum_{l=1}^{d} \int_{s-\epsilon}^{s} d r \mid\left\langle S(s-r, y-*)\left((\lambda-\mu)^{T} \cdot \sigma(u(r, y))\right)_{l},\right. \\
& \left.S(t-r, x-*)\left(\mu^{T} \cdot \sigma(u(r, x))\right)_{l}\right\rangle_{\mathscr{H}} \mid .
\end{aligned}
$$

Hypothesis $\mathbf{P 2}$ and [6, Lemma 6.1] together imply that

$$
\tilde{A}_{1} \geq c\|\lambda-\mu\| \epsilon^{\frac{2-\beta}{2}} .
$$

From (5.19) and (5.38), since $\|\lambda\|^{2}+\|\mu\|^{2}=1$, we see that

$$
J_{1}+J_{2} \geq c_{0} \epsilon^{\frac{2-\beta}{2}}-\frac{4}{3} \tilde{B}_{4}-8\left(J_{2}^{(2)}+J_{2}^{(3)}+A_{2}+A_{3}+A_{4}+A_{5}\right) .
$$

We have bounded the two terms $J_{2}^{(2)}$ and $J_{2}^{(3)}$ in (5.20) and (5.21). We now estimate the other five terms on the right-hand side of (5.39). As for the term $J_{2}^{(2)}$ and (5.20), we obtain that, for any $q \geq 1$,

$$
\mathrm{E}\left[\sup _{\|\xi\|=1}\left|A_{2}\right|^{q}\right] \leq c \epsilon^{(2-\beta) q} .
$$

Similar to $J_{2}^{(3)}$ and (5.21), we have for any $q \geq 1$,

$$
\mathrm{E}\left[\sup _{\|\xi\|=1}\left|A_{4}\right|^{q}\right] \leq c \epsilon^{(2-\beta) q} .
$$

Again, by [6, Lemma 6.2] with $a=1$, for any $q \geq 1$,

$$
\mathrm{E}\left[\sup _{\|\xi\|=1}\left|A_{5}\right|^{q}\right] \leq c(t-s+\epsilon)^{\frac{2-\beta}{2} q} \epsilon^{\frac{2-\beta}{2} q}
$$


Optimal lower bounds on hitting probabilities

$$
\leq c\left(\delta^{-\gamma_{0}} \epsilon^{\gamma_{0}}+\epsilon\right)^{\frac{2-\beta}{2} q} \epsilon^{\frac{2-\beta}{2} q} \leq c^{\prime} \epsilon^{\frac{2-\beta}{2}\left(1+\gamma_{0}\right) q},
$$

where, in the second inequality, we have used the assumption $t-s \leq \delta^{-\gamma_{0}} \epsilon^{\gamma_{0}}$.

We next bound the $q$-th moment of $A_{3}$. This is similar to the calculation in [6, p. 129], but with their exponent $\gamma$ replaced by $2-\beta$ since now we use our Theorem 1.1 instead of their (2.6). Hence, $\mathrm{E}\left[\sup _{\|\xi\|=1}\left|A_{3}\right|^{q}\right] \leq c a_{1} \times a_{2}$, where $a_{1}$ and $a_{2}$ are defined in [6, p. 129], that is,

$$
\begin{gathered}
a_{1}=\left(\int_{s-\epsilon}^{s} d r \int_{\mathbb{R}^{k}} d v \int_{\mathbb{R}^{k}} d z\|v-z\|^{-\beta} S(t-r, x-v) S(t-r, x-z)\right)^{q-1}, \\
a_{2}=\int_{s-\epsilon}^{s} d r \int_{\mathbb{R}^{k}} d v \int_{\mathbb{R}^{k}} d z\|v-z\|^{-\beta} S(t-r, x-v) \\
\times S(t-r, x-z)\|v-x\|^{\frac{2-\beta}{2} q}\|z-x\|^{\frac{2-\beta}{2} q}
\end{gathered}
$$

By [6, Lemma 6.1],

$$
\begin{aligned}
a_{1} & =c\left((t-s+\epsilon)^{\frac{2-\beta}{2}}-(t-s)^{\frac{2-\beta}{2}}\right)^{q-1} \\
& \leq c(t-s+\epsilon)^{\frac{2-\beta}{2}(q-1)} \leq c\left(\delta^{-\gamma_{0}} \epsilon^{\gamma_{0}}+\epsilon\right)^{\frac{2-\beta}{2}(q-1)} \\
& \leq c \epsilon^{\frac{2-\beta}{2}} \gamma_{0}(q-1)
\end{aligned}
$$

where, in the second inequality, we use the assumption $t-s \leq \delta^{-\gamma_{0}} \epsilon^{\gamma_{0}}$. For $a_{2}$, as in [6, p. 129], we use the change of variables $\tilde{v}=\frac{x-v}{\sqrt{t-r}}, \tilde{z}=\frac{x-z}{\sqrt{t-r}}$, to see that

$$
\begin{aligned}
a_{2} & =\int_{s-\epsilon}^{s} d r(t-r)^{\frac{(2-\beta) q}{2}-\frac{\beta}{2}} \int_{\mathbb{R}^{k}} d \tilde{v} \int_{\mathbb{R}^{k}} d \tilde{z} S(1, \tilde{v}) S(1, \tilde{z})\|\tilde{v}-\tilde{z}\|^{-\beta}\|\tilde{v}\|^{\frac{(2-\beta) q}{2}}\|\tilde{z}\|^{\frac{(2-\beta) q}{2}} \\
& =c\left((t-s+\epsilon)^{\frac{2-\beta}{2}(1+q)}-(t-s)^{\frac{2-\beta}{2}(1+q)}\right) \\
& \leq c\left(\delta^{-\gamma_{0}} \epsilon^{\gamma_{0}}+\epsilon\right)^{\frac{2-\beta}{2}(1+q)} \leq c^{\prime} \epsilon^{\frac{2-\beta}{2} \gamma_{0}(1+q)}
\end{aligned}
$$

where, in the first inequality, we use the assumption $t-s \leq \delta^{-\gamma_{0}} \epsilon^{\gamma_{0}}$. Therefore, from (5.45) and (5.46), we obtain

$$
\mathrm{E}\left[\sup _{\|\xi\|=1}\left|A_{3}\right|^{q}\right] \leq c \epsilon^{\frac{2-\beta}{2} \gamma_{0}(q-1)+\frac{2-\beta}{2} \gamma_{0}(1+q)}=c \epsilon^{(2-\beta) \gamma_{0} q} .
$$

We proceed to study the term $\tilde{B}_{4}$. Following the calculation in [6, p. 130], by hypothesis $\mathbf{P 1}$ and the semigroup property of $S(t, v)$,

$$
\begin{aligned}
\tilde{B}_{4} & \leq \tilde{c} \int_{0}^{\epsilon} d r \int_{\mathbb{R}^{k}} d v\|v\|^{-\beta}(t-s+2 r)^{-k / 2} \exp \left(-\frac{\|y-x+v\|^{2}}{2(t-s+2 r)}\right) \\
& \leq \tilde{c}\left(I_{1}+I_{2}\right),
\end{aligned}
$$

where

$$
\begin{aligned}
& I_{1}=\int_{0}^{\epsilon} d r \int_{\|v\| \leq \theta_{0} \sqrt{r}} d v\|v\|^{-\beta}(t-s+2 r)^{-k / 2} \exp \left(-\frac{\|y-x+v\|^{2}}{2(t-s+2 r)}\right), \\
& I_{2}=\int_{0}^{\epsilon} d r \int_{\|v\|>\theta_{0} \sqrt{r}} d v\|v\|^{-\beta}(t-s+2 r)^{-k / 2} \exp \left(-\frac{\|y-x+v\|^{2}}{2(t-s+2 r)}\right) .
\end{aligned}
$$

The constant $\theta_{0}$ above is a fixed and sufficiently large constant such that

$$
\frac{2 \tilde{c}}{2-\beta} \theta_{0}^{-\beta}<\frac{3 c_{0}}{8}
$$


where $c_{0}$ and $\tilde{c}$ are the constants in (5.39) and (5.48). By the choice of $\theta_{0}$, we have

$$
\begin{aligned}
\tilde{c} I_{2} & \leq \tilde{c} \int_{0}^{\epsilon} d r \theta_{0}^{-\beta} r^{-\beta / 2} \int_{\mathbb{R}^{k}} d v(t-s+2 r)^{-k / 2} \exp \left(-\frac{\|y-x+v\|^{2}}{2(t-s+2 r)}\right) \\
& =\tilde{c} \int_{0}^{\epsilon} d r \theta_{0}^{-\beta} r^{-\beta / 2}=\frac{2 \tilde{c}}{2-\beta} \theta_{0}^{-\beta} \epsilon^{\frac{2-\beta}{2}} \leq \frac{3 c_{0}}{8} \epsilon^{\frac{2-\beta}{2}} .
\end{aligned}
$$

As for $I_{1}$, it is bounded above by

$$
\int_{0}^{\epsilon} d r(t-s+2 r)^{-k / 2} \int_{\|v\| \leq \theta_{0} \sqrt{r}} d v\|v\|^{-\beta},
$$

and the $d v$-integral is equal to $c_{k} \theta_{0}^{k-\beta} r^{(k-\beta) / 2}$, so

$$
\begin{aligned}
I_{1} & \leq c_{k} \theta_{0}^{k-\beta} \int_{0}^{\epsilon} d r r^{(k-\beta) / 2}(t-s+2 r)^{-k / 2} \\
& \leq c_{k} \theta_{0}^{k-\beta} \int_{0}^{\epsilon} d r(t-s+2 r)^{-\beta / 2} \\
& =c_{k} \theta_{0}^{k-\beta}(2-\beta)^{-1} \epsilon^{\frac{2-\beta}{2}}\left[(2+(t-s) / \epsilon)^{\frac{2-\beta}{2}}-((t-s) / \epsilon)^{\frac{2-\beta}{2}}\right] \\
& \leq c_{k} \theta_{0}^{k-\beta}(2-\beta)^{-1} \epsilon^{\frac{2-\beta}{2}}\left[\left(2+\delta^{-1}\right)^{\frac{2-\beta}{2}}-\left(\delta^{-1}\right)^{\frac{2-\beta}{2}}\right],
\end{aligned}
$$

where, in the last inequality, we use the assumption $\epsilon<\delta(t-s)$ and Lemma 5.1(a). Since $\lim _{\delta \rightarrow 0}\left(2+\delta^{-1}\right)^{\frac{2-\beta}{2}}-\left(\delta^{-1}\right)^{\frac{2-\beta}{2}}=0$, we choose $\delta$ sufficiently small such that

$$
\tilde{c} c_{k} \theta_{0}^{k-\beta}(2-\beta)^{-1}\left[\left(2+\delta^{-1}\right)^{\frac{2-\beta}{2}}-\left(\delta^{-1}\right)^{\frac{2-\beta}{2}}\right] \leq \frac{3 c_{0}}{16},
$$

where $\tilde{c}, c_{k}$ and $c_{0}$ are the constants in (5.48), (5.51) and (5.39).

From the estimates in (5.20), (5.21), (5.39)-(5.52), we conclude that in the subcase $\delta(t-s)^{1 / \gamma_{0}}<\epsilon<\delta(t-s)$,

$$
\inf _{\|\xi\|=1} \xi^{T} \gamma_{Z} \xi \geq \frac{c_{0}}{4} \epsilon^{\frac{2-\beta}{2}}-Z_{\epsilon}^{2}
$$

where $Z_{\epsilon}^{2}:=8 \sup _{\|\xi\|=1}\left(J_{2}^{(2)}+J_{2}^{(3)}+A_{2}+A_{3}+A_{4}+A_{5}\right)$ satisfies that, for any $q \geq 1$,

$$
\mathrm{E}\left[\left|Z_{\epsilon}^{2}\right|^{q}\right] \leq c \epsilon^{(2-\beta) \gamma_{0} q} .
$$

Therefore, in the Case 1, from (5.27) and (5.53), for $\epsilon<\delta(t-s)$,

$$
\inf _{\|\xi\|=1} \xi^{T} \gamma_{Z} \xi \geq c \epsilon^{\frac{2-\beta}{2}}-Z_{\epsilon},
$$

where, by (5.28) and (5.54), $Z_{\epsilon}:=Z_{\epsilon}^{1} 1_{\left\{0<\epsilon<\delta(t-s)^{\left.1 / \gamma_{0}\right\}}\right.}+Z_{\epsilon}^{2} 1_{\left\{\delta(t-s)^{\left.1 / \gamma_{0} \leq \epsilon<\delta(t-s)\right\}}\right.}$ satisfies that, for any $q \geq 1$,

$$
\mathrm{E}\left[\left|Z_{\epsilon}\right|^{q}\right] \leq c \epsilon^{\min \left\{(2-\beta) \gamma_{0}, 1-\frac{\beta \gamma_{0}}{2}\right\} q} .
$$

Since $\left.\gamma_{0} \in\right] \frac{1}{2}, 1\left[\right.$, it is clear that $\min \left\{(2-\beta) \gamma_{0}, 1-\beta \gamma_{0} / 2\right\}>\frac{2-\beta}{2}$. Therefore, we apply [5, Proposition 3.5] to find that

$$
\begin{aligned}
\mathrm{E}\left[\left(\inf _{\|\xi\|=1} \xi^{T} \gamma_{Z} \xi\right)^{-2 p d}\right] & \leq c(\delta(t-s))^{-2 p d \frac{2-\beta}{2}}=c^{\prime}(t-s)^{-2 p d \frac{2-\beta}{2}} \\
& \leq \tilde{c}\left[|t-s|^{\frac{2-\beta}{2}}+\|x-y\|^{2-\beta}\right]^{-2 p d}
\end{aligned}
$$

whence follows the result in the case that $\|x-y\|^{2} \leq t-s<1$. 
Case 2. Now we work on the second case where $\|x-y\|>0$ and $\|x-y\|^{2} \geq t-s \geq 0$. Let $\epsilon>0$ be such that $(1+\alpha) \epsilon^{1 / 2}<\frac{1}{2}\|x-y\|$, where $\alpha>0$ is large but fixed; its specific value will be decided on later (see the explanation for (5.67) and (5.69)).

From here on, Case 2 is divided into two further subcases.

Subcase A. Suppose, in addition, that $\epsilon \geq \delta(t-s)$, where $\delta$ is chosen as in Case 1. In this subcase, we find that

$$
\xi^{T} \gamma_{Z} \xi \geq J_{1}+\tilde{J}_{2},
$$

where

$$
\tilde{J}_{2}:=\int_{(t-\epsilon) \vee s}^{t} d r \sum_{l=1}^{d}\|W\|_{\mathscr{H}}^{2}
$$

with $W$ as defined in (5.17), and $J_{1}$ has the same expression as in (5.15).

We estimate $J_{1}$ in the same way as in (5.29), i.e.,

$$
J_{1} \geq \frac{2}{3} A_{1}-8\left(A_{2}+A_{3}+A_{4}+A_{5}\right),
$$

where $A_{1}-A_{5}$ have the same expression as (5.30)-(5.34). Moreover, as in Subcase A of Case 1, we see that $A_{1} \geq \tilde{A}_{1}+\tilde{A}_{2}-2 \tilde{B}_{4}$, where $\tilde{A}_{1}, \tilde{A}_{2}$ and $\tilde{B}_{4}$ have the expressions as in (5.35)-(5.37).

As for $\tilde{J}_{2}$, we apply (5.18) and we subtract and add a "local" term to see that

$$
\tilde{J}_{2} \geq \frac{2}{3} B_{1}-4\left(B_{2}+B_{3}\right),
$$

where

$$
\begin{aligned}
B_{1} & =\sum_{l=1}^{d} \int_{(t-\epsilon) \vee s}^{t} d r\left\|S(t-r, x-*)\left(\mu^{T} \cdot \sigma(u(r, x))\right)_{l}\right\|_{\mathscr{H}}^{2}, \\
B_{2} & =\sum_{l=1}^{d} \int_{t-\epsilon}^{t} d r\left\|S(t-r, x-*)\left(\mu^{T} \cdot[\sigma(u(r, *))-\sigma(u(r, x))]\right)_{l}\right\|_{\mathscr{H}}^{2}, \\
B_{3} & =\int_{t-\epsilon}^{t} d r \sum_{l=1}^{d}\left\|\sum_{i=1}^{d} a_{i}(l, r, t, x) \mu_{i}\right\|_{\mathscr{H}}^{2} .
\end{aligned}
$$

By hypothesis $\mathbf{P 2}$ and [6, Lemma 6.1]

$$
\begin{aligned}
\tilde{A}_{2} & \geq c\|\mu\|^{2} \int_{s-\epsilon}^{s} d r\|S(t-r, x-*)\|_{\mathscr{H}}^{2} \\
& =c\|\mu\|^{2}\left((t-s+\epsilon)^{\frac{2-\beta}{2}}-(t-s)^{\frac{2-\beta}{2}}\right) .
\end{aligned}
$$

Similarly,

$$
\begin{aligned}
B_{1} & \geq c\|\mu\|^{2} \int_{(t-\epsilon) \vee s}^{t} d r\|S(t-r, x-*)\|_{\mathscr{H}}^{2} \\
& =c\|\mu\|^{2}(t-((t-\epsilon) \vee s))^{\frac{2-\beta}{2}}=c\|\mu\|^{2}((t-s) \wedge \epsilon)^{\frac{2-\beta}{2}} .
\end{aligned}
$$

From (5.38), (5.58) and (5.59), we see that

$$
\begin{aligned}
\tilde{A}_{1}+\tilde{A}_{2}+B_{1} & \geq c\left(\|\lambda-\mu\| \epsilon^{\frac{2-\beta}{2}}+\|\mu\|^{2}\left((t-s-\epsilon)^{\frac{2-\beta}{2}}-(t-s)^{\frac{2-\beta}{2}}+((t-s) \wedge \epsilon)^{\frac{2-\beta}{2}}\right)\right) \\
& =c \epsilon^{\frac{2-\beta}{2}}\left(\|\lambda-\mu\|+\|\mu\|^{2}\left(\left(\frac{t-s}{\epsilon}+1\right)^{\frac{2-\beta}{2}}-\left(\frac{t-s}{\epsilon}\right)^{\frac{2-\beta}{2}}+\left(\left(\frac{t-s}{\epsilon}\right) \wedge 1\right)^{\frac{2-\beta}{2}}\right)\right)
\end{aligned}
$$


Denote $\zeta(x):=(x+1)^{\frac{2-\beta}{2}}-x^{\frac{2-\beta}{2}}+(x \wedge 1)^{\frac{2-\beta}{2}}, x \in\left[0, \infty\left[\right.\right.$. Then it is clear that $\hat{c}_{0}:=$ $\min _{0 \leq x<\infty} \zeta(x)>0$. Thus we have

$$
\tilde{A}_{1}+\tilde{A}_{2}+B_{1} \geq c \epsilon^{\frac{2-\beta}{2}}\left(\|\lambda-\mu\|^{2}+\hat{c}_{0}\|\mu\|^{2}\right) \geq c^{\prime} \epsilon^{\frac{2-\beta}{2}} .
$$

The estimates of $A_{2}$ and $A_{4}$ in this subcase are the same as those in (5.40) and (5.41) respectively. Hence we have for any $q \geq 1$,

$$
\mathrm{E}\left[\sup _{\|\xi\|=1}\left|A_{2}\right|^{q}\right] \leq c \epsilon^{(2-\beta) q}, \quad \mathrm{E}\left[\sup _{\|\xi\|=1}\left|A_{4}\right|^{q}\right] \leq c \epsilon^{(2-\beta) q} .
$$

The term $B_{2}$ is similar to $J_{2}^{(2)}$ and $B_{3}$ is similar to $J_{2}^{(3)}$, so as in (5.20) and (5.21), for any $q \geq 1$,

$$
\mathrm{E}\left[\sup _{\|\xi\|=1}\left|B_{2}\right|^{q}\right] \leq c \epsilon^{(2-\beta) q}, \quad \mathrm{E}\left[\sup _{\|\xi\|=1}\left|B_{3}\right|^{q}\right] \leq c \epsilon^{(2-\beta) q} .
$$

Moreover, as in (5.42), by [6, Lemma 6.2] with $a=1$, for any $q \geq 1$,

$$
\begin{aligned}
\mathrm{E}\left[\sup _{\|\xi\|=1}\left|A_{5}\right|^{q}\right] & \leq c(t-s+\epsilon)^{\frac{2-\beta}{2} q} \epsilon^{\frac{2-\beta}{2} q} \\
& \leq c\left(\delta^{-1} \epsilon+\epsilon\right)^{\frac{2-\beta}{2} q} \epsilon^{\frac{2-\beta}{2} q}=c^{\prime} \epsilon^{(2-\beta) q},
\end{aligned}
$$

where, in the second inequality, we have used the assumption $t-s \leq \delta^{-1} \epsilon$.

We proceed to estimate the $q$-th moment of $A_{3}$. Again, we follow the calculation in [6, p. 129], use our Theorem 1.1 instead of their (2.6), to replace their exponent $\gamma$ by $2-\beta$. That is, $\mathrm{E}\left[\sup _{\|\xi\|=1}\left|A_{3}\right|^{q}\right] \leq c a_{1} \times a_{2}$, where $a_{1}$ and $a_{2}$ are defined in [6, p. 129] (see also our (5.43) and (5.44)). Similar to (5.45), we have, in this subcase $t-s \leq \delta^{-1} \epsilon$,

$$
\begin{aligned}
a_{1} & =c\left((t-s+\epsilon)^{\frac{2-\beta}{2}}-(t-s)^{\frac{2-\beta}{2}}\right)^{q-1} \\
& \leq c(t-s+\epsilon)^{\frac{2-\beta}{2}(q-1)} \leq c\left(\delta^{-1} \epsilon+\epsilon\right)^{\frac{2-\beta}{2}(q-1)}=c^{\prime} \epsilon^{\frac{2-\beta}{2}(q-1)} .
\end{aligned}
$$

Moreover, similar to (5.46),

$$
\begin{aligned}
a_{2} & =c\left((t-s+\epsilon)^{\frac{2-\beta}{2}(1+q)}-(t-s)^{\frac{2-\beta}{2}(1+q)}\right) \\
& \leq c\left(\delta^{-1} \epsilon+\epsilon\right)^{\frac{2-\beta}{2}(1+q)}=c^{\prime} \epsilon^{\frac{2-\beta}{2}(1+q)},
\end{aligned}
$$

where, in the inequality, we use the assumption $t-s \leq \delta^{-1} \epsilon$. Therefore,

$$
\mathrm{E}\left[\sup _{\|\xi\|=1}\left|A_{3}\right|^{q}\right] \leq c \epsilon^{\frac{2-\beta}{2}(q-1)+\frac{2-\beta}{2}(1+q)}=c \epsilon^{(2-\beta) q} .
$$

Furthermore, under the assumption $(1+\alpha) \epsilon^{1 / 2}<\frac{1}{2}\|x-y\|$, the estimate of $\tilde{B}_{4}$ follows exactly the same lines as in [6, pp. 130-131]. Indeed, by hypothesis $\mathbf{P 1}$ and using change of variable,

$$
\tilde{B}_{4} \leq c\left(I_{1}+I_{2}\right),
$$

where

$$
\begin{aligned}
& I_{1}=\int_{0}^{\epsilon} d r \int_{\|v\|<\sqrt{r}(1+\alpha)} d v\|v\|^{-\beta}(t-s+2 r)^{-k / 2} \exp \left(-\frac{\|y-x+v\|^{2}}{2(t-s+2 r)}\right), \\
& I_{2}=\int_{0}^{\epsilon} d r \int_{\|v\| \geq \sqrt{r}(1+\alpha)} d v\|v\|^{-\beta}(t-s+2 r)^{-k / 2} \exp \left(-\frac{\|y-x+v\|^{2}}{2(t-s+2 r)}\right) .
\end{aligned}
$$


Using the change of variables $\rho=\frac{t-s+2 r}{\alpha^{2} \epsilon}$ and the inequality $t-s \leq \delta^{-1} \epsilon$, we follow the calculation in [6, pp. 130-131] to see that, under the assumption $(1+\alpha) \epsilon^{1 / 2}<\frac{1}{2}\|x-y\|$,

$$
I_{1} \leq \epsilon^{\frac{2-\beta}{2}} \Phi_{1}(\alpha)
$$

where $\lim _{\alpha \rightarrow \infty} \Phi_{1}(\alpha)=0$. The estimate of $I_{2}$ is the same as that in [6, p. 131] and we have

$$
I_{2} \leq c(1+\alpha)^{-\beta} \epsilon^{\frac{2-\beta}{2}} .
$$

We note that $\lim _{\alpha \rightarrow \infty}(1+\alpha)^{-\beta}=0$ and so we have shown that

$$
\tilde{B}_{4} \leq \Phi(\alpha) \epsilon^{\frac{2-\beta}{2}}, \text { with } \lim _{\alpha \rightarrow \infty} \Phi(\alpha)=0 .
$$

Therefore, from (5.60) and (5.67), we have shown that, in the subcase $\epsilon \geq \delta(t-s)$,

$$
\inf _{\|\xi\|=1} \xi^{T} \gamma_{Z} \xi \geq c^{\prime} \epsilon^{\frac{2-\beta}{2}}-\frac{4}{3} \Phi(\alpha) \epsilon^{\frac{2-\beta}{2}}-\tilde{Z}_{\epsilon},
$$

where by (5.61)-(5.66), $\tilde{Z}_{\epsilon}:=\sup _{\|\xi\|=1} 8\left(A_{2}+A_{3}+A_{4}+A_{5}+B_{2}+B_{3}\right)$ satisfies that, for any $q \geq 1$,

$$
\mathrm{E}\left[\left|\tilde{Z}_{\epsilon}\right|^{q}\right] \leq c \epsilon^{(2-\beta) q} .
$$

We choose $\alpha$ large enough so that $\frac{4}{3} \Phi(\alpha)<\frac{c^{\prime}}{2}$, to get

$$
\inf _{\|\xi\|=1} \xi^{T} \gamma_{Z} \xi \geq \frac{c^{\prime}}{2} \epsilon^{\frac{2-\beta}{2}}-\tilde{Z}_{\epsilon}
$$

Subcase $B$. In this final subcase, we suppose that $\epsilon<\delta(t-s) \leq \delta\|x-y\|^{2}$. Choose and fix $0<\epsilon<\delta(t-s)$. During the course of our proof of Case 1, we established that

$$
\inf _{\|\xi\|=1} \xi^{T} \gamma_{Z} \xi \geq c \epsilon^{\frac{2-\beta}{2}}-Z_{\epsilon},
$$

where, for all $q \geq 1$,

$$
\mathrm{E}\left[\left|Z_{\epsilon}\right|^{q}\right] \leq c \epsilon^{\min \left\{(2-\beta) \gamma_{0}, 1-\frac{\beta \gamma_{0}}{2}\right\} q},
$$

with $\left.\gamma_{0} \in\right] 1 / 2,1[$ be a fixed constant (see (5.55) and (5.56)). This inequality remains valid in this Subcase B.

Combine Subcases A and B, and, in particular, (5.69) and (5.70), to find that for all $0<\epsilon<2^{-2}(1+\alpha)^{-2}\|x-y\|^{2}$,

$$
\inf _{\|\xi\|=1} \xi^{T} \gamma_{Z} \xi \geq c \epsilon^{\frac{2-\beta}{2}}-\left(Z_{\epsilon} 1_{\{\epsilon<\delta(t-s)\}}+\tilde{Z}_{\epsilon} 1_{\left\{t-s \leq \delta^{-1} \epsilon\right\}}\right) .
$$

Because of this and (5.56) and (5.68), by [5, Proposition 3.5], this implies that

$$
\begin{aligned}
\mathrm{E}\left[\left(\inf _{\|\xi\|=1} \xi^{T} \gamma_{Z} \xi\right)^{-2 p d}\right] & \leq c\|x-y\|^{2(-2 d p)\left(\frac{2-\beta}{2}\right)} \\
& \leq c\left(\|x-y\|^{2}+|t-s|\right)^{\left(\frac{2-\beta}{2}\right)(-2 d p)} \\
& \leq c\left(|t-s|^{\frac{2-\beta}{2}}+\|x-y\|^{2-\beta}\right)^{-2 d p} .
\end{aligned}
$$

This completes the proof of Theorem 1.3. 


\section{A Proof of $(5.1)$}

Proof of (5.1). By (2.1),

$$
\begin{aligned}
\mathrm{E} & {\left[\left\|D_{\cdot, *}^{(l)}\left(u_{i}(\rho, x)\right)\right\|_{\mathscr{H}_{s-\epsilon, s}}^{2 q}\right]=\mathrm{E}\left[\left\|D_{\cdot, *}^{(l)}\left(u_{i}(\rho, x)\right)\right\|_{\mathscr{H}_{s-\epsilon, s \wedge \rho}^{2 q}}\right] } \\
\leq & c \mathrm{E}\left[\left(\int_{s-\epsilon}^{s \wedge \rho} d r\left\|\sigma_{i l}(u(r, *)) S(\rho-r, x-*)\right\|_{\mathscr{H}^{\prime}}^{2}\right)^{q}\right] \\
& +c \mathrm{E}\left[\left\|\int_{s-\epsilon}^{\rho} \int_{\mathbb{R}^{k}} S(\rho-\theta, x-\eta) 1_{\{\theta>\cdot\}} \sum_{j=1}^{d} D_{\cdot, *}^{(l)}\left(\sigma_{i j}(u(\theta, \eta))\right) M^{j}(d \theta, d \eta)\right\|_{\mathscr{H}_{s-\epsilon, s \wedge \rho}}^{2 q}\right] \\
& +c \mathrm{E}\left[\left\|\int_{s-\epsilon}^{\rho} \int_{\mathbb{R}^{k}} S(\rho-\theta, x-\eta) 1_{\{\theta>\cdot\}} D_{\cdot, *}^{(l)}\left(b_{i}(u(\theta, \eta))\right) d \theta d \eta\right\|_{\mathscr{H}_{s-\epsilon, s \wedge \rho}}^{2 q}\right] \\
= & : A+B+C .
\end{aligned}
$$

By hypothesis P1, it is clear that

$$
A \leq c\left(\int_{s-\epsilon}^{s \wedge \rho} d \theta \int_{\mathbb{R}^{k}} \mu(d \xi)|\mathscr{F} S(\rho-\theta, *)(\xi)|^{2}\right)^{q} .
$$

Using Burkholder's inequality for Hilbert-space-valued martingales ([10, E.2. p. 212]) and hypothesis P1, we see that

$$
\begin{gathered}
B \leq c \sum_{j=1}^{d} \mathrm{E}\left[\left(\int_{s-\epsilon}^{\rho} d \theta \int_{\mathbb{R}^{k}} d z \int_{\mathbb{R}^{k}} d v\|z-v\|^{-\beta} S(\rho-\theta, x-z)\left\|D_{\cdot, *} u_{j}(\theta, z)\right\|_{\mathscr{H}_{s-\epsilon, s \wedge \theta}^{d}}\right.\right. \\
\left.\left.\times S(\rho-\theta, x-v)\left\|D_{\cdot, *} u_{j}(\theta, v)\right\|_{\mathscr{H}_{s-\epsilon, s \wedge \theta}^{d}}\right)^{q}\right]
\end{gathered}
$$

Moreover, using hypothesis P1,

$$
C \leq c \sum_{j=1}^{d} \mathrm{E}\left[\left(\int_{s-\epsilon}^{\rho} d \theta \int_{\mathbb{R}^{k}} d \eta S(\rho-\theta, x-\eta)\left\|D_{\cdot, *} u_{j}(\theta, \eta)\right\|_{\mathscr{H}_{s-\epsilon, s \wedge \theta}^{d}}\right)^{2 q}\right] .
$$

Case 1: $\rho \leq s$. Applying Minkowski's inequality with respect to the measure $\| z-$ $v \|^{-\beta} S(\rho-\theta, x-z) S(\rho-\theta, x-v) d v d z d \theta$,

$$
\begin{aligned}
B \leq c & \sum_{j=1}^{d} \sup _{(t, x) \in[0, T] \times \mathbb{R}^{k}} \mathrm{E}\left[\left\|D\left(u_{j}(t, x)\right)\right\|_{\mathscr{H}_{T}^{d}}^{2 q}\right] \\
& \times\left(\int_{s-\epsilon}^{\rho} d \theta \int_{\mathbb{R}^{k}} d z \int_{\mathbb{R}^{k}} d v\|z-v\|^{-\beta} S(\rho-\theta, x-z) S(\rho-\theta, x-v)\right)^{q} \\
\leq & \left(\int_{s-\epsilon}^{\rho} d \theta \int_{\mathbb{R}^{k}} \mu(d \xi)|\mathscr{F} S(\rho-\theta, *)(\xi)|^{2}\right)^{q},
\end{aligned}
$$

where the second inequality holds by (2.3). Similarly, using Minkowski's inequality with respect to the measure $S(\rho-\theta, x-\eta) d \theta d \eta$,

$$
\begin{aligned}
C & \leq c \sum_{j=1}^{d} \sup _{(t, x) \in[0, T] \times \mathbb{R}^{k}} \mathrm{E}\left[\left\|D\left(u_{j}(t, x)\right)\right\|_{\mathscr{H}_{T}^{d}}^{2 q}\right]\left(\int_{s-\epsilon}^{\rho} d \theta \int_{\mathbb{R}^{k}} d \eta S(\rho-\theta, x-\eta)\right)^{2 q} \\
& \leq c(\rho-s+\epsilon)^{2 q} \leq c^{\prime}(\rho-s+\epsilon)^{(2-\beta) q / 2} \\
& =c^{\prime \prime}\left(\int_{s-\epsilon}^{\rho} d \theta \int_{\mathbb{R}^{k}} \mu(d \xi)|\mathscr{F} S(\rho-\theta, *)(\xi)|^{2}\right)^{q},
\end{aligned}
$$

where the second inequality holds by (2.3) and the equality follows from [6, Lemma 6.1]. 
Optimal lower bounds on hitting probabilities

Hence, in Case 1, (A.2), (A.5) and (A.6) prove (5.1).

Case 2: $\rho>s$. Let $a_{1}=s-\epsilon, b_{1}=s, a_{2}=s, b_{2}=\rho$.

From (A.3), we bound $B$ by $B_{1}+B_{2}$, where for $i=1,2$,

$$
\begin{gathered}
B_{i} \leq c \sum_{j=1}^{d} \mathrm{E}\left[\left(\int_{a_{i}}^{b_{i}} d \theta \int_{\mathbb{R}^{k}} d z \int_{\mathbb{R}^{k}} d v\|z-v\|^{-\beta} S(\rho-\theta, x-z)\left\|D_{\cdot, *} u_{j}(\theta, z)\right\|_{\mathscr{H}_{s-\epsilon, s \wedge \theta}^{d}}\right.\right. \\
\left.\left.\times S(\rho-\theta, x-v)\left\|D_{\cdot, *} u_{j}(\theta, v)\right\|_{\mathscr{H}_{s-\epsilon, s \wedge \theta}^{d}}\right)^{q}\right] .
\end{gathered}
$$

From the calculation in (A.5), we have

$$
B_{1} \leq c\left(\int_{s-\epsilon}^{s} d \theta \int_{\mathbb{R}^{k}} \mu(d \xi)|\mathscr{F} S(\rho-\theta, *)(\xi)|^{2}\right)^{q} .
$$

Applying Hölder's inequality with respect to the measure $\|z-v\|^{-\beta} S(\rho-\theta, x-z) S(\rho-$ $\theta, x-v) d v d z d \theta$ and the Cauchy-Schwartz inequality,

$$
\begin{gathered}
B_{2} \leq c \sum_{j=1}^{d} \int_{s}^{\rho} d \theta \int_{\mathbb{R}^{k}} d z \int_{\mathbb{R}^{k}} d v\|z-v\|^{-\beta} S(\rho-\theta, x-z) S(\rho-\theta, x-v) \\
\quad \times \sup _{x \in \mathbb{R}^{k}} \mathrm{E}\left[\left\|D_{\cdot, *} u_{j}(\theta, x)\right\|_{\mathscr{H}_{s-\epsilon, s \wedge \theta}^{d}}^{2 q}\right] \\
=c \sum_{j=1}^{d} \int_{s}^{\rho} d \theta(\rho-\theta)^{-\frac{\beta}{2}} \sup _{x \in \mathbb{R}^{k}} \mathrm{E}\left[\left\|D_{\cdot, *} u_{j}(\theta, x)\right\|_{\mathscr{H}_{s-\epsilon, s}^{d}}^{2 q}\right],
\end{gathered}
$$

where the equality follows from $[6,(6.3)]$.

Moreover, from (A.4), we bound $C$ by $C_{1}+C_{2}$, where for $i=1,2$,

$$
C_{i} \leq c \sum_{j=1}^{d} \mathrm{E}\left[\left(\int_{a_{i}}^{b_{i}} d \theta \int_{\mathbb{R}^{k}} d \eta S(\rho-\theta, x-\eta)\left\|D_{\cdot, *} u_{j}(\theta, \eta)\right\|_{\mathscr{H}_{s-\epsilon, s \wedge \theta}^{d}}\right)^{2 q}\right] .
$$

Similar to the derivation of the first inequality in (A.6), we see that

$$
\begin{aligned}
C_{1} & \leq c\left(\int_{s-\epsilon}^{s} d \theta \int_{\mathbb{R}^{k}} d \eta S(\rho-\theta, x-\eta)\right)^{2 q}=c \epsilon^{2 q} \\
& \leq c^{\prime}\left((\rho-s+\epsilon)^{\frac{2-\beta}{2}}-(\rho-s)^{\frac{2-\beta}{2}}\right)^{q} \\
& =c^{\prime \prime}\left(\int_{s-\epsilon}^{s} d \theta \int_{\mathbb{R}^{k}} \mu(d \xi)|\mathscr{F} S(\rho-\theta, *)(\xi)|^{2}\right)^{q},
\end{aligned}
$$

where the second inequality holds since by Lemma 5.1(a), $(\rho-s+\epsilon)^{\frac{2-\beta}{2}}-(\rho-s)^{\frac{2-\beta}{2}} \geq$ $(T+\epsilon)^{\frac{2-\beta}{2}}-T^{\frac{2-\beta}{2}} \geq C \epsilon$ for all $\epsilon \in[0, T]$, and the second equality follows from [6, Lemma 6.1]. Applying Hölder's inequality with respect to the measure $S(\rho-\theta, x-\eta) d \theta d \theta$ and the Cauchy-Schwartz inequality,

$$
\begin{aligned}
C_{2} & \leq c \sum_{j=1}^{d} \int_{s}^{\rho} d \theta \int_{\mathbb{R}^{k}} d \eta S(\rho-\theta, x-\eta) \sup _{x \in \mathbb{R}^{k}} \mathrm{E}\left[\left\|D_{\cdot, *} u_{j}(\theta, x)\right\|_{\mathscr{H}_{s-\epsilon, s \wedge \theta}^{d}}^{2 q}\right] \\
& =c \int_{s}^{\rho} d \theta \sum_{j=1}^{d} \sup _{x \in \mathbb{R}^{k}} \mathrm{E}\left[\left\|D_{\cdot, *} u_{j}(\theta, x)\right\|_{\mathscr{H}_{s-\epsilon, s}^{d}}^{2 q}\right] .
\end{aligned}
$$

Denote

$$
\varphi(\theta)=\sum_{j=1}^{d} \sup _{x \in \mathbb{R}^{k}} \mathrm{E}\left[\left\|D_{\cdot, *} u_{j}(\theta, x)\right\|_{\mathscr{H}_{s-\epsilon, s}^{d}}^{2 q}\right] .
$$


We deduce, from (A.1), (A.2) and (A.7)-(A.10), that

$$
\varphi(\rho) \leq c\left(\int_{s-\epsilon}^{s} d \theta \int_{\mathbb{R}^{k}} \mu(d \xi)|\mathscr{F} S(\rho-\theta, *)(\xi)|^{2}\right)^{q}+c \int_{s}^{\rho}\left(1+(\rho-\theta)^{-\beta / 2}\right) \varphi(\theta) d \theta .
$$

By Gronwall's lemma ([2, Lemma 15]), we conclude that for $\rho \geq s$,

$$
\varphi(\rho) \leq c\left(\int_{s-\epsilon}^{s} d \theta \int_{\mathbb{R}^{k}} \mu(d \xi)|\mathscr{F} S(\rho-\theta, *)(\xi)|^{2}\right)^{q},
$$

which implies (5.1).

\section{References}

[1] Conus, D., Joseph, M., Khoshnevisan, D. and Shiu, S.: On the chaotic character of the stochastic heat equation, II. Probab. Theory Related Fields 156, (2013), 483-533. MR3078278

[2] Dalang, R.C.: Extending the martingale measure stochastic integral with application to spatially homogeneous S.P.D.E's. Electon. J. Probab. 4, (1999), 1-29. MR-1684157

[3] Dalang, R.C. and Frangos, N.E.: The stochastic wave equation in two spatial dimensions. Ann. Probab. 26, (1998), 187-212. MR-1617046

[4] Dalang, R.C., Khoshnevisan, D. and Nualart, E.: Hitting probabilities for systems of non-linear stochastic heat equations with additive noise. ALEA 3, (2007), 231-271. MR-2365643

[5] Dalang, R.C., Khoshnevisan, D. and Nualart, E.: Hitting probabilities for systems of non-linear stochastic heat equations with multiplicative noise. Probab. Th. Rel. Fields 144, (2009), 371-424. MR-2496438

[6] Dalang, R.C., Khoshnevisan, D. and Nualart, E.: Hitting probabilities for systems of non-linear stochastic heat equations in spatial dimension $k \geq 1$. Stoch PDE: Anal Comp 1, (2013), 94-151. MR-3327503

[7] Dalang, R.C. and Pu, F.: Optimal lower bounds on hitting probabilities for non-linear systems of stochastic fractional heat equations (2018). arXiv:1810.05386. MR-3828166

[8] Hu, Y., Nualart, D. and Song, J.: A nonlinear stochastic heat equation: Hölder continuity and smoothness of the density of the solution. Stochastic Process. Appl. 123, (2013), 1083-1103. MR-3005015

[9] Li, K.: Hölder continuity for stochastic fractional heat equation with colored noise. Statist. Probab. Lett. 129, (2017), 34-41. MR-3688512

[10] Métivier, M.: Semimartingales. de Gruyter, 1982. MR-0688144

[11] Nualart, D.: The Malliavin Calculus and Related Topics (2nd ed). Springer, London, 2006. MR-2200233

[12] Nualart, D.: Analysis on Wiener space and anticipating stochastic calculus. In: Ecole d'Eté de Probabilités de Saint-Flour XXV. Lect. Notes in Math. 1690, 123-227. Springer, Heidelberg, 1998. MR-1668111

[13] Nualart, D. and Quer-Sardanyons, L.: Existence and smoothness of the density for spatially homogeneous SPDEs. Potential Anal. 27, (2007), 281-299. MR-2336301

[14] Revuz, D. and Yor, M.: Continuous Martingales and Brownian Motion (3rd ed). SpringerVerlag, 1999. MR-1725357

[15] Saint Loubert Bié, E.: Étude d'une EDPS conduite par un bruit poissonnien. Probab. Th. Rel. Fields 111, (1998), 287-321. MR-1633586

[16] Sanz-Solé, M.: Malliavin Calculus with Applications to Stochastic Partial Differential Equations. EPFL Press, Lausanne, 2005. MR-2167213

[17] Sanz-Solé, M. and Sarrà, M.: Hölder continuity for the stochastic heat equation with spatially correlated noise. In: Seminar on Stochastic Analysis, Random Fields and Applications, III (Ascona, 1999). Progr. Probab. 52, 259-268. Birkhäuser, Basel, 2002. MR-1958822 
Optimal lower bounds on hitting probabilities

[18] Walsh, J.B.: An introduction to stochastic partial differential equations. In: Ecole d'Eté de Probabilités de Saint-Flour XIV. Lect. Notes in Math. 1180, 266-437. Springer, Heidelberg, 1986. MR-0876085

[19] Watanabe, S.: Lectures on stochastic differential equations and Malliavin calculus. In: Tata Institute of Fundamental Research Lectures on Mathematics and Physics 73. Springer, Berlin, 1984. MR-0742628 


\section{Electronic Journal of Probability Electronic Communications in Probability}

\section{Advantages of publishing in EJP-ECP}

- Very high standards

- Free for authors, free for readers

- Quick publication (no backlog)

- Secure publication $\left(\mathrm{LOCKSS}^{1}\right)$

- Easy interface (EJMS²)

\section{Economical model of EJP-ECP}

- Non profit, sponsored by $\mathrm{IMS}^{3}, \mathrm{BS}^{4}$, ProjectEuclid ${ }^{5}$

- Purely electronic

\section{Help keep the journal free and vigorous}

- Donate to the IMS open access fund ${ }^{6}$ (click here to donate!)

- Submit your best articles to EJP-ECP

- Choose EJP-ECP over for-profit journals

\footnotetext{
${ }^{1}$ LOCKSS: Lots of Copies Keep Stuff Safe http://www. lockss.org/

${ }^{2}$ EJMS: Electronic Journal Management System http://www.vtex.lt/en/ejms.html

${ }^{3}$ IMS: Institute of Mathematical Statistics http://www.imstat.org/

${ }^{4}$ BS: Bernoulli Society http://www. bernoulli-society.org/

${ }^{5}$ Project Euclid: https://projecteuclid.org/

${ }^{6}$ IMS Open Access Fund: http://www.imstat.org/publications/open.htm
} 